\title{
On absolute moments of characteristic polynomials of a certain class of complex random matrices
}

\author{
Yan V. Fyodorov* and Boris A. Khoruzhenko ${ }^{\dagger}$
}

31 January 2006

\begin{abstract}
Integer moments of the spectral determinant $|\operatorname{det}(z I-W)|^{2}$ of complex random matrices $W$ are obtained in terms of the characteristic polynomial of the Hermitian matrix $W W^{*}$ for the class of matrices $W=A U$ where $A$ is a given matrix and $U$ is random unitary. This work is motivated by studies of complex eigenvalues of random matrices and potential applications of the obtained results in this context are discussed.
\end{abstract}

\section{Introduction}

Characteristic polynomials of random matrices have recently attracted considerable interest in the mathematical physics literature. Initially, the interest was stimulated by applications in number theory [35, 36], quantum chaos [3, 21, 27] and quantum chromodynamics (QCD) [42, 45, 29, 22, but with the emerging connections to integrable systems [39, 46], combinatorics [16, 43, representation theory [9, 10, 12, 15] and analysis [5], it has become apparent that characteristic polynomials of random matrices are also of independent interest.

In this paper we are concerned with integer moments of the squared modulus of characteristic polynomial of complex random matrices in a rather general class of matrices $W=A U$, where $A$ a given positive semidefinite Hermitian matrix, $A \geq 0$, and $U$ is a random unitary matrix distributed uniformly over the unitary group.

In the particular case when $A$ is identity matrix, the matrix $W$ is random unitary, and its eigenvalues lie on the unit circle. Various moments of the characteristic polynomial for this class of matrices were obtained recently, see [35, 36, 13, 14, 15. In the general

${ }^{*}$ School of Mathematical Sciences, University of Nottingham, Nottingham, NG7 2RD, U.K.; Email: yan.fyodorov@nottingham.ac.uk. The research in Nottingham is supported by EPSRC grant EP/C515056/1: "Random Matrices and Polynomials: a tool to understand complexity"

†School of Mathematical Sciences, Queen Mary, University of London, London E1 4NS, U.K.; E-mail: b.khoruzhenko@qmul.ac.uk. A significant part of this work was carried out during the Newton Institute programme on Random Matrix Approaches in Number Theory (26 January-16 July 2004) 
case, the eigenvalues of $W=A U$ will be distributed in a region in the complex plane. Eigenvalue statistics of such complex eigenvalues, and in particular the mean eigenvalue density, are of interest for physics of open chaotic systems, see, e.g. 24, 25], and in QCD, see, e.g. [46] and references therein, and are difficult to study analytically. In this context moments of the squared modulus of the characteristic polynomial frequently provide a very useful tool. Indeed, in a variety of random matrix ensembles the mean eigenvalue density,

$$
\rho(x, y)=\langle\operatorname{tr} \delta(z I-W)\rangle_{W}, \quad z=x+i y,
$$

can be expressed in terms of the mean-square-modulus of the characteristic polynomial in a closely related random matrix ensemble. In (1.1) the angle brackets stand for averaging over the matrix distribution, and $I$ is identity matrix.

An obvious example is served by the Ginibre ensemble of complex matrices [31. In this ensemble the matrix distribution has density Const. $\times \exp \left(-\operatorname{tr} W W^{*}\right)$ where $W^{*}$ is complex conjugate transpose of $W$. The mean density $\rho_{n}(x, y)$ of eigenvalues of Ginibre matrices of size $n \times n$ is given by

$$
\rho_{n}(x, y)=\frac{1}{\pi} e^{-|z|^{2}} \sum_{k=0}^{n-1} \frac{|z|^{2 k}}{k !} .
$$

One can arrive at (1.2) in various ways. Ginibre computed the joint probability density function of all eigenvalues and then applied the method of orthogonal polynomials. Another way is to use the method of dimensional reduction, see e.g. 44, 17, 18, which yields the following relation

$$
\rho_{n}(x, y)=\frac{e^{-|z|^{2}}}{\pi(n-1) !}\left\langle\left|\operatorname{det}\left(z I_{n-1}-W_{n-1}\right)\right|^{2}\right\rangle_{W_{n-1}} .
$$

Here the angle brackets stand for averaging over the Ginibre ensemble of complex matrices of size $(n-1) \times(n-1)$. The mean-square on the r.h.s. in (1.3) can be easily computed yielding again (1.2).

A less obvious example, which in fact provided the initial impetus for the present study, is the so-called ensemble of 'random contractions' 25. In its simplest variant of rank-one deviations from the unitarity, these are random $n \times n$ matrices satisfying the constraint

$$
W_{n} W_{n}^{*}=\left(\begin{array}{cc}
1-\gamma & 0 \\
0 & I_{n-1}
\end{array}\right), \quad 0<\gamma<1
$$

In the 'polar' coordinates, $W_{n}=G_{n} U_{n}$ where $U_{n}$ is a $\mathrm{CUE}_{n}$ matrix, i.e. a matrix drawn at random from the unitary group $U(n)$, and $G_{n}=\operatorname{diag}(\sqrt{1-\gamma}, 1, \ldots, 1)$. The mean density of eigenvalues ${ }^{1}$ of $W_{n}$ can be expressed as the mean square modulus of the characteristic polynomial of $(n-1) \times(n-1)$ matrices $\tilde{G}_{n-1} U_{n-1}$,

$$
\rho_{n}(x, y)=\frac{n-1}{\pi \gamma|z|^{2}}\left(\frac{\tilde{\gamma}}{\gamma}\right)^{n-2}\left\langle\left|\operatorname{det}\left(z I_{n-1}-\tilde{G}_{n-1} U_{n-1}\right)\right|^{2}\right\rangle_{U_{n-1}}, \quad 1-\gamma<|z|^{2}<1,
$$

\footnotetext{
${ }^{1}$ Note that constraint (1.4) implies that the eigenvalues of $W$ lie in the annulus $1-\gamma \leq|z|^{2} \leq 1$.
} 
where now the angle brackets stand for averaging over the unitary group $U(n-1)$ with respect to the normalized Haar measure, and

$$
\tilde{G}_{n-1}=\operatorname{diag}(\sqrt{1-\tilde{\gamma}}, 1, \ldots, 1), \quad \tilde{\gamma}=\frac{|z|^{2}+\gamma-1}{|z|^{2}} .
$$

Another example is provided by finite-rank deviations from Hermiticity [23]. We only consider the simplest but still non-trivial case of rank-one deviations. Let

$$
W_{n}=H_{n}+i \Gamma_{n}
$$

where $H_{n}$ is a $\mathrm{GUE}_{n}$ matrix, i.e. random Hermitian matrix of size $n \times n$ with probability distribution

$$
d P_{\beta, n}(H)=\text { Const. } \times e^{-\frac{\beta}{2} \operatorname{tr} H^{2}} \prod_{j=1}^{n} d H_{j j} \prod_{1 \leq j<k \leq n} \frac{d H_{j j} d \overline{H_{j j}}}{2}, \quad \beta>0,
$$

and $\Gamma_{n}=\operatorname{diag}(\gamma, 0, \ldots, 0), \gamma>0$. It is apparent that all eigenvalues of $W$ lie in the strip $0 \leq y \leq \gamma$. For the mean eigenvalue density $\rho_{n}(x, y)$ of $W_{n}$ we have

$$
\rho_{n}(x, y)=r_{\beta, n}(x, y)\langle| \operatorname{det}\left(z I_{n-1}-\left.\left(H_{n-1}+i \tilde{\Gamma}_{n-1}\right)\right|^{2}\right\rangle_{H_{n-1}}, \quad 0<y<\gamma,
$$

where

$$
r_{\beta, n}(x, y)=\frac{\beta^{n}(\gamma-y)^{n-2} e^{-\frac{\beta x^{2}}{2}-\beta(\gamma-y) y}}{4 \sqrt{2 \pi \beta} \gamma^{n-1}(n-2) !}, \quad \tilde{\Gamma}_{n-1}=\operatorname{diag}(\gamma-y, 0, \ldots, 0),
$$

and the angle brackets stand for averaging with respect to the distribution $d P_{\beta, n-1}(H)$. We derive (1.5) and (1.7) in Section 6]

The above formulas relating the mean eigenvalue density and the mean-square-modulus of characteristic polynomial are specific to the considered matrix distributions. In general situation, the mean density of eigenvalues can be determined from fractional moments of the squared modulus $|\operatorname{det}(z I-W)|^{2}=\operatorname{det}(z I-W)(z I-W)^{*}$ (e.g., by the way of the logarithmic potential of the eigenvalue distribution), or from averages of ratios of $\operatorname{det}\left[(z I-W)(z I-W)^{*}+\varepsilon^{2} I\right]$, see e.g. [25]. Getting explicit formulas for that kind of objects outside the classes of Hermitian and unitary matrices is, however, a considerable challenge. Although it is known that the fractional moments of $|\operatorname{det}(z I-W)|^{2}$ can be written in terms of a hypergeometric function of matrix argument $W W^{*}$ [39], the corresponding series is hard to deal with in the limit of infinite matrix dimension.

Our main result, Theorem 11, expresses

$$
\left\langle\left[\operatorname{det}(z I-A U)(z I-A U)^{*}\right]^{ \pm m}\right\rangle_{U}, \quad m=1,2, \ldots,
$$

where the integration is over unitary matrices $U$ with respect to the Haar measure, as an $m$-fold integral of powers of the characteristic polynomial of the (Hermitian!) matrix $A A^{*}$. This integral can be written an $m \times m$ determinant with entries given by a certain integral 
transform of the characteristic polynomial of $A A^{*}$, see (2.11)-(2.12). In particular, this result implies that for the ensembles of random complex matrices $W$ with unitary invariant matrix distribution (for example, for the Feinberg-Zee ensemble [19] whose probability density of matrix entries of $W$ depends only on $W W^{*}$ ) our formulas effectively reduce the original non-Hermitian problem to a Hermitian one, albeit on the level of characteristic polynomials. This, as explained in more detail at the end of the next section, has a clear computational advantage, as one can then use various formulas for averages of products and ratios of characteristic polynomials of Hermitian matrices which have been obtained in recent years, see [11, 26, 5]. In contrast, with the exception of essentially Gaussian weights [1, 2], no such formulas are known for complex matrices.

We also express

$$
\left\langle\frac{1}{\operatorname{det}\left[(z I-A U)(z I-A U)^{*}+\varepsilon^{2} I\right]}\right\rangle_{U}
$$

as a two-fold integral of the inverse spectral determinant of $A A^{*}$, see Theorem 2 Again, the non-Hermitian problem is reduced to a Hermitian one. This regularized inverse spectral determinant can be useful as an indicator of the domain of the distribution of complex eigenvalues. For, any point $z$ where the above average blows up in the limit $\varepsilon \rightarrow 0$ must belong to the domain of eigenvalue distribution.

Acknowledgments. We would like to thank A. Gamburd for useful discussions and, in particular, for bringing reference 44 to our attention and for pointing us towards the link between our Lemma 3 and the Selberg Integral. We are also grateful to Ph. Biane for bringing reference [28] to our attention.

\section{Statement of main results and discussion}

Let $n$ and $m$ be positive integers. Define

$$
d \mu_{n}\left(t_{1}, \ldots, t_{m}\right)=\frac{1}{c_{n}} \Delta^{2}\left(t_{1}, \ldots, t_{m}\right) \prod_{j=1}^{m}\left(1+t_{j}\right)^{-n-2 m} d t_{1} \ldots d t_{m}, \quad t_{j} \geq 0,
$$

and, for $n \geq 2 m$,

$$
d \nu_{n}\left(t_{1}, \ldots, t_{m}\right)=\frac{1}{k_{n}} \Delta^{2}\left(t_{1}, \ldots, t_{m}\right) \prod_{j=1}^{m}\left(1-t_{j}\right)^{n-2 m} d t_{1} \ldots d t_{m}, \quad 0 \leq t_{j} \leq 1,
$$

where

$$
\Delta\left(t_{1}, \ldots, t_{m}\right)=\operatorname{det}\left(t_{i}^{m-j}\right)_{i, j=1}^{m}=\prod_{1 \leq i<j \leq m}\left(t_{i}-t_{j}\right)
$$

is the Vardermonde determinant, and

$$
c_{n}=\prod_{j=0}^{m-1} \frac{j !(j+1) !(n+j) !}{(n+m+j) !} \quad \text { and } \quad k_{n}=\prod_{j=0}^{m-1} \frac{j !(j+1) !(n-m-j-1) !}{(n-j-1) !}
$$


are the normalization constants. The Selberg Integral, see e.g. [38], asserts that $d \mu_{n}$ and $d \nu_{n}$ are unit mass measures,

$$
\int_{0}^{\infty} \ldots \int_{0}^{\infty} d \mu_{n}\left(t_{1}, \ldots, t_{m}\right)=\int_{0}^{1} \ldots \int_{0}^{1} d \nu_{n}\left(t_{1}, \ldots, t_{m}\right)=1
$$

The measures $d \mu_{n}$ and $d \nu_{n}$ define probability distributions which have the following random matrix interpretation. Consider two families of matrix distributions on the space of $m \times m$ complex matrices $Z=\left(x_{j k}+i y_{j k}\right)_{j, k=1}^{m}$ :

$$
d \hat{\mu}_{n}(Z)=\frac{1}{\hat{c}_{n}} \frac{1}{\operatorname{det}^{n+2 m}\left(I_{m}+Z Z^{*}\right)} \prod_{j, k=1}^{m} d x_{j k} d y_{j k}, \quad n \geq 0
$$

and

$$
d \hat{\nu}_{n}(Z)=\frac{1}{\hat{k}_{n}} \operatorname{det}^{n-2 m}\left(I_{m}-Z Z^{*}\right) \prod_{j, k=1}^{m} d x_{j k} d y_{j k}, \quad n \geq 2 m .
$$

The measures $d \hat{\nu}_{n}(Z)$ are defined on the matrix ball $Z Z^{*}<I_{m}$ and the constants $\hat{c}_{n}$ and $\hat{k}_{n}$ are determined by the normalization condition

$$
\int_{Z Z^{*} \geq 0} d \hat{\mu}_{n}(Z)=\int_{0 \leq Z Z^{*} \leq I_{m}} d \hat{\nu}_{n}(Z)=1
$$

A standard calculation, see e.g. [30], shows that if $s\left(Z Z^{*}\right)$ is a symmetric function of the eigenvalues $t_{1}, \ldots, t_{m}$ of $Z Z^{*}$, i.e. $s\left(Z Z^{*}\right)=s\left(t_{1}, \ldots, t_{m}\right)$, then

$$
\begin{aligned}
\int_{Z Z^{*} \geq 0} s\left(Z Z^{*}\right) d \hat{\mu}_{n}(Z) & =\int_{0}^{+\infty} \ldots \int_{0}^{+\infty} s\left(t_{1}, \ldots, t_{m}\right) d \mu_{n}\left(t_{1}, \ldots t_{m}\right), \\
\int_{0 \leq Z Z^{*} \leq I_{m}} s\left(Z Z^{*}\right) d \hat{\nu}_{n}(Z) & =\int_{0}^{1} \ldots \int_{0}^{1} s\left(t_{1}, \ldots, t_{m}\right) d \nu_{n}\left(t_{1}, \ldots t_{m}\right) .
\end{aligned}
$$

Theorem 1 below, which we state in a slightly more generality than required for spectral determinants, tells how to integrate moments of determinants over the unitary group equipped with the Haar measure $d U$ fixed by the normalization $\int_{U(n)} d U=1$.

Theorem 1 Let $A, B, C, D$ be complex matrices of size $n \times n$.

(i) For any positive integer $m$

$$
\int_{U(n)} \operatorname{det}^{m}\left[(A U+C)(B U+D)^{*}\right] d U=\int_{0}^{\infty} \ldots \int_{0}^{\infty} \prod_{j=1}^{m} \operatorname{det}\left(C D^{*}+t_{j} A B^{*}\right) d \mu_{n}\left(t_{1}, \ldots, t_{m}\right)
$$


(ii) If $A A^{*}<C C^{*}$ and $B B^{*}<D D^{*}$ then for any positive integer $m$ such that $2 m \leq n$

$$
\int_{U(n)} \frac{d U}{\operatorname{det}^{m}\left[(A U+C)(B U+D)^{*}\right]}=\int_{0}^{1} \ldots \int_{0}^{1} \frac{d \nu_{n}\left(t_{1}, \ldots, t_{m}\right)}{\prod_{j=1}^{m} \operatorname{det}\left(C D^{*}-t_{j} A B^{*}\right)} .
$$

Remark. Identities (2.9) - (2.10) may be written in yet another form by making use of the well-known identity

$$
\int \ldots \int \operatorname{det}\left(p_{j}\left(t_{i}\right)\right)_{i, j=1}^{m} \operatorname{det}\left(q_{j}\left(t_{i}\right)\right)_{i, j=1}^{m} d t_{1} \ldots d t_{m}=m ! \operatorname{det}\left(\int p_{i}(t) q_{j}(t) d t\right)_{i, j=1}^{m} .
$$

We have

$$
\int_{U(n)} \operatorname{det}^{m}\left[(A U+C)(B U+D)^{*}\right] d U=\frac{m !}{c_{n}} \operatorname{det}\left(\int_{0}^{+\infty} \frac{\operatorname{det}\left(C D^{*}+t A B^{*}\right) t^{i+j} d t}{(1+t)^{n+2 m}}\right)_{i, j=0}^{m-1}
$$

and

$$
\int_{U(n)} \frac{d U}{\operatorname{det}^{m}\left[(A U+C)(B U+D)^{*}\right]}=\frac{m !}{k_{n}} \operatorname{det}\left(\int_{0}^{1} \frac{(1-t)^{n-2 m} t^{i+j} d t}{\operatorname{det}\left(C D^{*}-t A B^{*}\right)}\right)_{i, j=0}^{m-1} .
$$

Obviously, by letting $C=D=z I$ in (2.9) and (2.10) one obtains formulas for moments of the spectral determinants $|\operatorname{det}(z I-A U)|^{2}$. In particular,

$$
\int_{U(n)}|\operatorname{det}(z I-A U)|^{2} d U=(n+1) \int_{0}^{\infty} \frac{\operatorname{det}\left(|z|^{2} I+t A A^{*}\right)}{(1+t)^{n+2}} d t
$$

and, provided $n \geq 2$,

$$
\int_{U(n)} \frac{d U}{|\operatorname{det}(z I-A U)|^{2}}= \begin{cases}\int_{0}^{1} \frac{(n-1)(1-t)^{n-2}}{\operatorname{det}\left(A A^{*}-t|z|^{2} I\right)} d t, & \text { if }|z|^{2}<\lambda_{\min }\left(A A^{*}\right) \\ \int_{0}^{1} \frac{(n-1)(1-t)^{n-2}}{\operatorname{det}\left(|z|^{2} I-t A A^{*}\right)} d t, & \text { if }|z|^{2}>\lambda_{\max }\left(A A^{*}\right)\end{cases}
$$

where $\lambda_{\min }\left(A A^{*}\right)$ and $\lambda_{\max }\left(A A^{*}\right)$ are respectively the smallest and largest eigenvalues of $A A^{*}$.

If $\lambda_{\min }\left(A A^{*}\right) \leq|z|^{2} \leq \lambda_{\max }\left(A A^{*}\right)$, then the integral on the left-hand side in (2.14) should be handled with care. One way to do this is to regularize the integrand. 
For positive $\varepsilon$, define

$$
R_{z, \varepsilon}\left(A, A^{*}\right)=\int_{U(n)} \frac{d U}{\operatorname{det}\left[\varepsilon^{2} I+\left(I-\frac{1}{z} A U\right)\left(I-\frac{1}{z} A U\right)^{*}\right]} .
$$

The integral on the right-hand side is, in fact, a function of $A A^{*}$ and our next theorem evaluates this function in terms of the eigenvalues of $A A^{*}$.

Theorem 2 Let $\varepsilon>0$, and assume that $n \geq 2$. Then for any $n \times n$ matrix $A$ and any non-zero complex z

$$
R_{z, \varepsilon}\left(A, A^{*}\right)=\frac{n-1}{2 \pi i} \int_{0}^{1}(1-t)^{n-2} d t \int_{-\infty}^{+\infty} \frac{d x}{x} \frac{1}{\operatorname{det}\left[\frac{1}{|z|^{2}} A A^{*}+\left(\varepsilon^{2}-t\right) I-i \varepsilon \sqrt{t}\left(x+\frac{1}{x}\right) I\right]} .
$$

If the eigenvalues $a_{j}^{2}$ of $A A^{*}$ are all distinct, then for any non-zero $z$ in the annulus $\lambda_{\min }\left(A A^{*}\right)<|z|^{2}<\lambda_{\max }\left(A A^{*}\right)$ we have

$$
\lim _{\varepsilon \rightarrow 0} \frac{R_{z, \varepsilon}\left(A, A^{*}\right)}{\ln \left(1 / \varepsilon^{2}\right)}=(n-1)|z|^{2} \sum_{j=1}^{n}\left(|z|^{2}-a_{j}^{2}\right)^{n-2} \theta\left(|z|^{2}-a_{j}^{2}\right) \prod_{k \neq j} \frac{1}{a_{k}^{2}-a_{j}^{2}},
$$

where $\theta$ is the Heaviside step function, $\theta(r)=1 / 2$ at $r=0$.

We prove Theorems 1 and 2 in Sections 4 and 5, respectively, by making use of two techniques, which to a certain extent are equivalent. One is based on the expansion of moments of spectral determinants in characters of the unitary group and subsequent use of the orthogonality of characters. On this way, Theorem 1 is equivalent to two combinatorial identities (3.18) and (3.19), one of which is a particular case of the Selberg integral in the form of Kaneko 34 and Kadell 33. We prove (3.18) and (3.19) in Section 3. These combinatorial identities can be stated in the form of matrix integrals (3.28) and (3.29) and are of independent interest. They lead to evaluation of some non-trivial matrix integrals, as discussed at the end of Section 3. The other technique is based on the so-called color-flavor transformation, due to Zirnbauer [48]. This transformation has many uses, and in the random-matrix context it provides a very convenient tool to handle moments of spectral determinants.

As an application of Theorem 11, let us consider random matrices (1.4). In the limit $n \rightarrow \infty$ the eigenvalues of $W_{n}$ get closer and closer to the unit circle. Let $\mathcal{N}_{n}(a, b)$ be the number of eigenvalues of $W_{n}$ in the annulus

$$
D_{a, b}=\left\{z: \frac{2 a}{n} \leq 1-|z|^{2} \leq \frac{2 b}{n}\right\}, \quad 0<a<b .
$$

By (1.5),

$$
\left\langle\mathcal{N}_{n}(a, b)\right\rangle_{U(n)}=\int_{D_{a, b}}\left(\frac{n-1}{\pi \gamma|z|^{2}}\left(\frac{\tilde{\gamma}}{\gamma}\right)^{n-2} \int_{U(n-1)}\left|\operatorname{det}\left(z I_{n-1}-\tilde{G}_{n-1} U_{n-1}\right)\right|^{2} d U_{n-1}\right) d x d y .
$$


Making use of (2.13),

$$
\int_{U(n-1)}\left|\operatorname{det}\left(z I_{n-1}-\tilde{G}_{n-1} U_{n-1}\right)\right|^{2} d U_{n-1}=n \int_{0}^{\infty} \frac{\left[|z|^{2}+t(1-\tilde{\gamma})\right]\left(|z|^{2}+t\right)^{n-2}}{(1+t)^{n+1}} d t
$$

and

where

$$
\frac{1}{n}\left\langle\mathcal{N}_{n}(a, b)\right\rangle_{U(n)}=\pi \int_{1-\frac{2 b}{n}}^{1-\frac{2 a}{n}} f_{n}(q) d q
$$

$$
f_{n}(q)=\frac{n-1}{\pi \gamma q}\left(\frac{\tilde{\gamma}}{\gamma}\right)^{n-2} \int_{0}^{\infty} \frac{[q+t(1-\tilde{\gamma})](q+t)^{n-2}}{(1+t)^{n+1}} d t
$$

and $\tilde{\gamma}=\left(|z|^{2}+\gamma-1\right) /|z|^{2}$. Letting $n \rightarrow \infty$, we obtain, after simple manipulations, that

$$
\lim _{n \rightarrow \infty} \frac{1}{n}\left\langle\mathcal{N}_{n}(a, b)\right\rangle_{U(n)}=\frac{\sinh a}{a} \exp \left(\frac{a(\gamma-2)}{\gamma}\right)-\frac{\sinh b}{b} \exp \left(\frac{b(\gamma-2)}{\gamma}\right),
$$

recovering one of the formulas of [25], who, using a different method requiring knowledge of the joint probability distribution of eigenvalues, found the mean density of eigenvalues and higher order correlation functions for the general case of finite-rank deviation from the CUE. Note that when $\gamma=1$ the nonzero eigenvalues of $G_{n} U_{n}$ coincide with the $(n-1) \times(n-1)$ matrix obtained from $U_{n}$ by removing its first row and column, see [49] for more information about eigenvalue statistics of truncated unitary matrices.

Now, we would like to elaborate on the point made at the end of Introduction. Consider random complex matrices $W$ of the size $n \times n$ with unitary invariant matrix distribution. Then, by making use of the unitary invariance and Theorem 1

$$
\left\langle|\operatorname{det}(z I-W)|^{2}\right\rangle_{W}=\left\langle\int_{U(n)}|\operatorname{det}(z I-W U)|^{2} d U\right\rangle_{W}=\int_{0}^{\infty} \frac{p_{n}\left(|z|^{2} t\right)}{(1+t)^{n+2}} d t,
$$

where

$$
p_{n}(x)=\left\langle\operatorname{det}\left(x I+W W^{*}\right)\right\rangle_{W} .
$$

A similar formula holds for higher order moments of $|\operatorname{det}(z I-W)|^{2}$. Thus, Theorem 1 reduces the original non-Hermitian problem to a Hermitian one.

The integral on the right-hand side in (2.16) can be evaluated, in the limit of infinite matrix dimension, in terms of the limiting eigenvalue distribution of the non-negative matrices $W W^{*}$. To this end, consider, for example, the complex $n \times n$ matrices $W$ with the matrix distribution characterized by the Feinberg-Zee density

$$
\text { Const. } \times e^{-n \operatorname{tr} V\left(W W^{*}\right)},
$$

where $V(r)$ is a polynomial in $r, V(r)=a_{m} r^{m}+\ldots, a_{m}>0$. Then

$$
p_{n}(x)=e^{n \int \ln (x+\lambda) d w(\lambda)}(1+o(1)),
$$


where $d w(\lambda)$ is the limiting normalised eigenvalue counting measure of $W W^{*}$, and it can be shown that

$$
\lim _{n \rightarrow \infty} \frac{1}{n} \ln \left\langle|\operatorname{det}(z I-W)|^{2}\right\rangle_{W}=\Phi(x, y),
$$

where

$$
\Phi(x, y)= \begin{cases}\ln |z|^{2} & \text { if }|z|>m_{1}=\int \lambda d w(\lambda) \\ \int_{0}^{\infty} \ln \lambda d w(\lambda) & \text { if } \frac{1}{|z|}>m_{-1}=\int \frac{d w(\lambda)}{\lambda} \\ |z|^{2}+\int_{0}^{\infty} \ln \frac{\lambda+t_{0}}{|z|^{2}+t_{0}} d w(\lambda) & \text { if } 1 / m_{-1}<|z|<m_{1}\end{cases}
$$

where $t_{0}$ is the unique non-negative solution of

$$
\int_{0}^{\infty} \frac{d w(\lambda)}{\lambda+t}=\frac{1}{|z|^{2}+t}
$$

The function $\Phi(x, y)$ is subharmonic and, hence, defines a measure $d \nu=\frac{1}{4 \pi} \Delta \Phi$ in the complex plane. Here $\Delta$ is the Laplacian in variables $x$ and $y$. For the Ginibre ensemble of random matrices this measure can be found explicitly. In this case $V(r)=r$ and $W W^{*}$ is a Wishart ensemble of random matrices. Its limiting eigenvalue distribution $d w(\lambda)$ is given by $d w(\lambda)=\frac{1}{2 \pi} \sqrt{(4-\lambda) / \lambda}, \quad 0<\lambda<4$, with $m_{1}=1$ and $m_{-1}=\infty$. A straightforward but tedious calculation shows that $\Phi(x, y)=|z|^{2}-1$ inside the unit disk $|z|^{2}<1$. Therefore $d \nu$ is the uniform distribution on the unit disk, which is the same as the limiting eigenvalue distribution in the Ginibre ensemble of random matrices, and, hence, for this ensemble

$$
\lim _{n \rightarrow \infty} \frac{1}{n}\left\langle\ln |\operatorname{det}(z I-W)|^{2}\right\rangle_{W}=\lim _{n \rightarrow \infty} \frac{1}{n} \ln \left\langle|\operatorname{det}(z I-W)|^{2}\right\rangle_{W},
$$

so that the operations of taking logarithm and taking average commute in the limit $n \rightarrow \infty$. A similar relation is known to hold for Wigner ensembles of Hermitian matrices [6].

It would be interesting to investigate conditions on random matrix distributions which guarantee (2.19). As the left hand-side in (2.19) is the logarithmic potential of the limiting eigenvalue distribution of $W$, this together with our Theorem 1 would give a useful tool for calculating eigenvalue distributions in the complex plane. There are indications that the range of matrix distributions for which (2.19) holds is quite wide and contains the invariant ensembles (2.17). Indeed, $\Phi(x, y)$ of equation (2.18) reproduces the density of eigenvalue distribution in ensembles (2.17) which was obtained in [19, 20] with the help of the method of Hermitization ${ }^{2}$. In this context we would like to mention calculation of Brown's measure for $R$-diagonal elements in finite von Neumann algebras [28], see also [8] .

\footnotetext{
${ }^{2}$ This method has a hidden regularization procedure which has to justified to satisfy the mathematical rigor.
} 
A matrix model for such elements is provided by random matrices $R U$ where $U$ is random unitary and $R$ is positive-definite, and Brown's measure is in a way a regularized version of the eigenvalue distribution. Again, $\Phi(x, y)$ of equation (2.18) reproduces Brown's measure found in 28 .

\section{Combinatorial identities}

Schur functions. In order to make our paper self-contained we recall below the required facts from the theory of symmetric polynomials.

A partition is a finite sequence $\lambda=\left(\lambda_{1}, \lambda_{2}, \ldots, \lambda_{n}\right)$ of integers, called parts, such that $\lambda_{1} \geq \lambda_{2} \geq \ldots \geq \lambda_{n} \geq 0$. The weight of a partition, $|\lambda|$, is the sum of its parts $|\lambda|=\sum_{j} \lambda_{j}$, and the length, $l(\lambda)$, is the number of its non-zero parts. No distinction is made between partitions which differ merely by the number of zero parts, and different partitions of weight $r$ represent different ways to write $r$ as a sum of natural numbers.

Partitions can be viewed as Young diagrams. The Young diagram of $\lambda$ is a rectangular array of boxes (or dots), with $\lambda_{j}$ boxes in the $j$ th row, the rows being lined up on the left. By transposing the diagram of $\lambda$ (i.e. interchanging the rows and columns) one obtains another partition. This partition is called conjugate of $\lambda$ and denoted by $\lambda^{\prime}$. For example the conjugate of the partition $(r)$ of length one is the partition $(1, \ldots, 1) \equiv\left(1^{r}\right)$ of length $r$. Obviously, $l\left(\lambda^{\prime}\right)=\lambda_{1}$ and $|\lambda|=\left|\lambda^{\prime}\right|$.

For any partition $\lambda$ of length $l(\lambda) \leq n$

$$
s_{\lambda}\left(x_{1}, \ldots, x_{n}\right)=\frac{\operatorname{det}\left(x_{i}^{\lambda_{j}+n-j}\right)_{i, j=1}^{n}}{\operatorname{det}\left(x_{i}^{n-j}\right)_{i, j=1}^{n}}
$$

is a symmetric polynomial in $x_{1}, \ldots, x_{n}$, homogeneous of degree $|\lambda|$. These polynomials are known as the Schur functions. By convention, $s_{\lambda}\left(x_{1}, \ldots, x_{n}\right)=0$ if $l(\lambda)>n$. This convention is in agreement with the apparent identities

$$
\begin{aligned}
s_{\lambda}\left(x_{1}, \ldots, x_{n-1}, 0\right) & =s_{\lambda}\left(x_{1}, \ldots, x_{n-1}\right) & & \text { if } l(\lambda) \leq n-1 \\
& =0 & & \text { if } l(\lambda)>n-1 .
\end{aligned}
$$

For partitions of length one, $\lambda=(r)$, the Schur functions $s_{\lambda}$ are the complete symmetric functions $h_{r}$,

$$
s_{(r)}\left(x_{1}, \ldots, x_{n}\right)=h_{r}\left(x_{1}, \ldots, x_{n}\right)=\sum_{1 \leq i_{1} \leq i_{2} \leq \ldots \leq i_{r} \leq n} x_{i_{1}} x_{i_{2}} \ldots x_{i_{r}},
$$

and $s_{\lambda^{\prime}}$ are the elementary symmetric functions $e_{r}$,

$$
s_{\left(1^{r}\right)}\left(x_{1}, \ldots, x_{n}\right)=e_{r}\left(x_{1}, \ldots, x_{n}\right)=\sum_{1 \leq i_{1}<i_{2}<\ldots<i_{r} \leq n} x_{i_{1}} x_{i_{2}} \ldots x_{i_{r}} .
$$


More generally, see e.g. 37] p. 41, the Jacobi-Trudi identity asserts that for any $n \geq l(\lambda)$,

$$
s_{\lambda}=\operatorname{det}\left(h_{\lambda_{i}-i+j}\right)_{i, j=1}^{n}, \quad s_{\lambda^{\prime}}=\operatorname{det}\left(e_{\lambda_{i}-i+j}\right)_{i, j=1}^{n}
$$

where, by convention, $e_{r}=h_{r}=0$ if $r<0$.

We shall also need the Schur functions of matrix argument. If $M$ is an $n \times n$ matrix then

$$
s_{\lambda}(M)=s_{\lambda}\left(x_{1}, \ldots, x_{n}\right)
$$

where $x_{1}, \ldots, x_{n}$ are the eigenvalues of $M$. Thus $s_{\lambda}(M)$ is a symmetric polynomial in the eigenvalues of $M$. In view of (3.6), it is also a polynomial in the matrix entries of $M$. The Schur functions of matrix argument are the characters of irreducible representations of the general linear group and its unitary subgroup and, as a consequence, have an important property of orthogonality. If $\lambda$ and $\mu$ are two partitions and $A$ and $B$ are two $n \times n$ matrices then, see e.g. [37] p. 445,

$$
\int_{U(n)} s_{\lambda}(A U) \overline{s_{\mu}(B U)} d U=\delta_{\lambda, \mu} \frac{s_{\lambda}\left(A B^{*}\right)}{d_{\lambda}},
$$

and

$$
\int_{U(n)} s_{\lambda}\left(A U B U^{*}\right) d U=\frac{s_{\lambda}(A) s_{\lambda}(B)}{d_{\lambda}},
$$

where $d_{\lambda}$ is the dimension of the irreducible representations of $U(n)$ with signature $\lambda$,

$$
d_{\lambda}=s_{\lambda}\left(I_{n}\right)=s_{\lambda}\left(1_{n}\right)
$$

We use the notation $\left(1_{n}\right)$ for the $n$-tuple $(1, \ldots, 1)$.

If $\lambda$ is a partition of length $1, \lambda=(r)$, then

$$
s_{\lambda}\left(1_{n}\right)=h_{r}\left(1_{n}\right)=\left(\begin{array}{c}
n+r-1 \\
r
\end{array}\right)=\frac{(n+r-1) !}{r !(n-1) !}
$$

and

$$
s_{\lambda^{\prime}}\left(1_{n}\right)=e_{r}\left(1_{n}\right)=\left(\begin{array}{c}
n \\
r
\end{array}\right)=\frac{n !}{r !(n-r) !} .
$$

In general, explicit expressions are known for $s_{\lambda}\left(1_{n}\right)$ and $s_{\lambda^{\prime}}\left(1_{n}\right)$ in terms of the $\lambda_{j}$ 's. If $l(\lambda) \leq n$ then for any $m \geq l(\lambda)$

$$
s_{\lambda}\left(1_{n}\right)=\left\{\prod_{1 \leq i<j \leq m}\left(\lambda_{i}-i-\lambda_{j}+j\right)\right\} \times \prod_{j=1}^{m} \frac{\left(n+\lambda_{j}-j\right) !}{\left(m+\lambda_{j}-j\right) !(n-j) !} .
$$

If $l\left(\lambda^{\prime}\right) \leq n$ then for any $m \geq l(\lambda)$

$$
s_{\lambda^{\prime}}\left(1_{n}\right)=\left\{\prod_{1 \leq i<j \leq m}\left(\lambda_{i}-i-\lambda_{j}+j\right)\right\} \times \prod_{j=1}^{m} \frac{(n+j-1) !}{\left(n+j-1-\lambda_{j}\right) !\left(m+\lambda_{j}-j\right) !} .
$$


Both identities can be derived by evaluating the binomial determinants in (3.6).

We shall also need the Cauchy identities for Schur functions, see, e.g., 37, pp. 63, 65. Let $X$ be an $n \times n$ matrix. Then

$$
\begin{aligned}
& \prod_{i=1}^{m} \operatorname{det}\left(I_{n}+t_{i} X\right)=\sum_{\lambda} s_{\lambda}\left(t_{1}, \ldots, t_{m}\right) s_{\lambda^{\prime}}(X) \\
& \prod_{i=1}^{m} \frac{1}{\operatorname{det}\left(I_{n}-t_{i} X\right)}=\sum_{\lambda} s_{\lambda}\left(t_{1}, \ldots, t_{m}\right) s_{\lambda}(X) .
\end{aligned}
$$

The summation in (3.13) is over all partitions such that $l(\lambda) \leq m$ and $l\left(\lambda^{\prime}\right) \leq n$ and is finite. The summation in (3.14) is over all partitions such that $l(\lambda) \leq \min (m, n)$ and is infinite. The corresponding series converges absolutely if $X X^{*}<I_{n}$.

Beta-function determinants. When $m=1$ identities (3.13) and (3.14) take the familiar form of the expansion of the characteristic polynomial and its reciprocal in terms of the elementary symmetric functions and complete symmetric functions, respectively. In this case Theorem 1 is a straightforward consequence of the orthogonality property of the Schur functions (3.7) and the Euler integral

$$
\int_{0}^{1} t^{p-1}(1-t)^{q-1} d t=\int_{0}^{+\infty} \frac{t^{p-1}}{(1+t)^{p+q}} d t=\frac{\Gamma(p) \Gamma(q)}{\Gamma(p+q)}=B(p, q), \quad \operatorname{Re} p, q>0
$$

where $\Gamma(p)$ and $B(p, q)$ are the Gamma and Beta functions respectively. Indeed, for example,

$$
\begin{aligned}
\int_{U(n)} \operatorname{det}(I+A U) \operatorname{det}(I+B U)^{*} d U & =\sum_{r=0}^{n} \frac{e_{r}\left(A B^{*}\right)}{e_{r}\left(1_{n}\right)} \\
& =(n+1) \int_{0}^{\infty} \operatorname{det}\left(I+t A B^{*}\right) \frac{d t}{(1+t)^{n+2}},
\end{aligned}
$$

where we have used (3.15) in the form

$$
\frac{1}{e_{r}\left(1_{n}\right)}=(n+1) \int_{0}^{+\infty} \frac{t^{r}}{(1+t)^{n+2}} d t .
$$

Similarly,

$$
\frac{1}{h_{r}\left(1_{n}\right)}=(n-1) \int_{0}^{1} t^{r}(1-t)^{n-2} d t
$$

and this identity does the trick for the reciprocal characteristic polynomials.

Our proof of Theorem 1 uses the following generalization of (3.16) - (3.17) to multivariate setting.

Lemma 3 Let $m$ and $n$ be nonnegative integers. 
(a) For any partition $\lambda$ such that $l(\lambda) \leq m$ and $l\left(\lambda^{\prime}\right) \leq n$

$$
\frac{s_{\lambda}^{2}\left(1_{m}\right)}{s_{\lambda^{\prime}}\left(1_{n}\right)}=\frac{1}{c_{n}} \int_{0}^{\infty} \ldots \int_{0}^{\infty} s_{\lambda}\left(t_{1}, \ldots t_{m}\right) \Delta^{2}\left(t_{1}, \ldots, t_{m}\right) \prod_{j=1}^{m} \frac{d t_{j}}{\left(1+t_{j}\right)^{n+2 m}} .
$$

(b) If $2 m \leq n$ then for any partition $\lambda$ such that $l(\lambda) \leq m$

$$
\frac{s_{\lambda}^{2}\left(1_{m}\right)}{s_{\lambda}\left(1_{n}\right)}=\frac{1}{k_{n}} \int_{0}^{1} \ldots \int_{0}^{1} s_{\lambda}\left(t_{1}, \ldots t_{m}\right) \Delta^{2}\left(t_{1}, \ldots, t_{m}\right) \prod_{j=1}^{m}\left(1-t_{j}\right)^{n-2 m} d t_{j} .
$$

The normalization constants $c_{n}$ and $k_{n}$ are given in (2.4), and $\Delta\left(t_{1}, \ldots, t_{m}\right)$ is the Vandermonde determinant (2.3).

Remark. Identity (3.19) can be inferred from a generalization of the Selberg Integral due to Kaneko [34] and Kadell [33], of which (3.19) is a particular case corresponding to a special choice of parameters. However, we are not aware about any generalization of the Selberg Integral leading to (3.18). Below, we give an elementary proof of (3.18) and (3.19) which is based on evaluating a determinant consisting of Beta functions, see Proposition 4 below. Our proof has a limited scope and does not extend to the generality of Kaneko and Kadell formulas.

Proof. Let $f_{j}=m+\lambda_{j}-j, j=1,2, \ldots, m$. If $l(\lambda) \leq m \leq n$ and $l\left(\lambda^{\prime}\right) \leq n$ then by (3.11) - (3.12)

$$
\frac{s_{\lambda}^{2}\left(1_{m}\right)}{s_{\lambda^{\prime}}\left(1_{n}\right)}=\Delta\left(f_{1}, \ldots, f_{m}\right) \times\left(\prod_{j=0}^{m-1} \frac{1}{j !^{2}(n+j) !}\right) \times\left(\prod_{j=1}^{m} f_{j} !\left(n+m-1-f_{j}\right) !\right)
$$

where

$$
\Delta\left(f_{1}, \ldots, f_{m}\right)=\prod_{1 \leq i<j \leq m}\left(f_{i}-f_{j}\right)=\operatorname{det}\left(f_{j}^{m-i}\right)_{i, j=1}^{m} .
$$

By adding rows in the Vandermonde determinant $\operatorname{det}\left(f_{j}^{m-i}\right)_{1}^{m}$,

$$
\Delta\left(f_{1}, \ldots, f_{m}\right)=\operatorname{det}\left(p_{m-i}\left(f_{j}\right)\right)_{i, j=1}^{m},
$$

where

$$
p_{k}(x)=(x+1)(x+2) \ldots(x+k) .
$$

Hence

$$
f_{1} ! f_{2} ! \ldots f_{m} ! \Delta\left(f_{1}, \ldots, f_{m}\right)=\operatorname{det}\left(\left(f_{j}+m-i\right) !\right)_{i, j=1}^{m}
$$

and

$$
\frac{s_{\lambda}^{2}\left(1_{m}\right)}{s_{\lambda^{\prime}}\left(1_{n}\right)}=\left(\prod_{j=0}^{m-1} \frac{(n+m+j) !}{(j !)^{2}(n+j) !}\right) \times \operatorname{det}\left(B\left(f_{j}+m-i+1, n+m-f_{j}\right)\right)_{i, j=1}^{m}
$$


where $B$ is the Beta function. By making use of Proposition 4 below,

$$
\begin{aligned}
\operatorname{det}\left(B\left(f_{j}+m-i+1, n+m-f_{j}\right)\right)_{i, j=1}^{m} & =\operatorname{det}\left(B\left(f_{j}+m-i+1, n+m-f_{j}+i-1\right)\right)_{i, j=1}^{m} \\
& =\operatorname{det}\left(\int_{0}^{+\infty} \frac{t^{f_{j}} t^{m-i} d t}{(1+t)^{n+2 m}}\right)_{i, j=1}^{m} .
\end{aligned}
$$

It is apparent that

$$
\begin{aligned}
\operatorname{det}\left(\int_{0}^{+\infty} \frac{t^{f_{j}} t^{m-i} d t}{(1+t)^{n+2 m}}\right)_{i, j=1}^{m} & =\int_{0}^{+\infty} \ldots \int_{0}^{+\infty} s_{\lambda}\left(t_{1}, \ldots t_{m}\right) \operatorname{det}\left(t_{i}^{m-j}\right)_{i, j=1}^{m} \prod_{i=1}^{m} \frac{t_{i}^{m-i} d t_{i}}{\left(1+t_{i}\right)^{n+2 m}} \\
& =\frac{1}{m !} \int_{0}^{+\infty} \ldots \int_{0}^{+\infty} s_{\lambda}\left(t_{1}, \ldots t_{m}\right)\left[\operatorname{det}\left(t_{i}^{m-j}\right)_{i, j=1}^{m}\right]^{2} \prod_{i=1}^{m} \frac{d t_{i}}{\left(1+t_{i}\right)^{n+2 m}},
\end{aligned}
$$

and (3.18) follows.

Similarly, if $2 m \leq n$ and $l(\lambda) \leq m$ then by (3.11)

$$
\frac{s_{\lambda}^{2}\left(1_{m}\right)}{s_{\lambda}\left(1_{n}\right)}=\Delta\left(f_{1}, \ldots, f_{m}\right) \times\left(\prod_{j=0}^{m-1} \frac{(n-j-1) !}{j !^{2}}\right) \times\left(\prod_{j=1}^{m} \frac{f_{j} !}{\left(n-m+f_{j}\right) !}\right),
$$

and, in view of (3.20),

$$
\frac{s_{\lambda}^{2}\left(1_{m}\right)}{s_{\lambda}\left(1_{n}\right)}=\left(\prod_{j=0}^{m-1} \frac{(n-j-1) !}{(j !)^{2}(n-m-j-1) !}\right) \times \operatorname{det}\left(B\left(f_{j}+m-i+1, n-2 m+i\right)\right)_{i, j=1}^{m}
$$

By Proposition 4 .

$$
\begin{aligned}
\operatorname{det}\left(B\left(f_{j}+m-i+1, n-2 m+i\right)\right)_{i, j=1}^{m} & =\operatorname{det}\left(B\left(f_{j}+m-i+1, n-2 m+1\right)\right)_{i, j=1}^{m} \\
& =\operatorname{det}\left(\int_{0}^{1} t^{f_{j}} t^{m-i}(1-t)^{n-2 m} d t\right)_{i, j=1}^{m},
\end{aligned}
$$

and (3.19) follows.

Proposition 4 For any $p_{1}, p_{2}, \ldots, p_{m}$ and $q_{1}, q_{2}, \ldots, q_{m}$ such that $\operatorname{Re} p_{j}>m$ and $\operatorname{Re} q_{j}>$ -1 we have

$$
\operatorname{det}\left(B\left(p_{j}-i, q_{j}+i\right)\right)_{1}^{m}=\operatorname{det}\left(B\left(p_{j}-i, q_{j}+1\right)\right)_{1}^{m}
$$

Proof. We shall use the identity

$$
B(p, q-1)+B(p-1, q)=B(p-1, q-1)
$$


and the operation of addition of columns to transform the determinant on the left in (3.24) to the one on the right.

It is convenient to write determinants by showing their columns. With this convention,

$$
|B(p-1, q+1), B(p-2, q+2), \ldots, B(p-m+1, q+m-1), B(p-m, q+m)|
$$

represent the determinant on the 1.h.s. in (3.24). Let us label its columns by numbers $1, \ldots, m$ from left to right (so that the leftmost column is column 1). Note a particular property of columns in this determinant. As we move from column $j$ to column $j+1$ the first argument of the Beta function decreases by one 1 the second argument increases by 1. To be able to refer to this property, we say that columns $1,2, \ldots, m$ are balanced.

Observing that column 1 has the desired form already, let us perform the following operation on columns $2,3, \ldots, m$. Starting at column $m$ and working backwards, let us add to each column the one that precedes it. In view of (3.25) and the above mentioned property of columns, this operation yields

$|B(p-1, q+1), B(p-2, q+1), B(p-3, q+2), \ldots, B(p-m+1, q+m-2), B(p-m, q+m-1)|$.

Observing that columns 1 and 2 have the desired form now, and that columns $2, \ldots, m$ remain balanced, we apply our operation again, now on columns $3, \ldots, m$. This yields the determinant

$|B(p-1, q+1), B(p-2, q+1), B(p-3, q+1), \ldots, B(p-m+1, q+m-3), B(p-m, q+m-2)|$,

where columns $1,2,3$ have the desired form and columns $4, \ldots, m$ are balanced. It is clear that repeated application of our operation will yield the determinant

$$
|B(p-1, q+1), B(p-2, q+1), B(p-3, q+1), \ldots, B(p-m, q+1)|
$$

after the final step. This is exactly the determinant on the right in (3.25).

Applications to matrix integrals. In view of integration formulas (2.7) and (2.8), identities (3.18) and (3.19) can be rewritten as:

$$
\int_{Z Z^{*} \geq 0} s_{\lambda}\left(Z Z^{*}\right) d \hat{\mu}_{n}(Z)=\frac{s_{\lambda}^{2}\left(I_{m}\right)}{s_{\lambda^{\prime}}\left(I_{n}\right)}, \quad \int_{Z Z^{*} \leq I_{m}} s_{\lambda}\left(Z Z^{*}\right) d \hat{\nu}_{n}(Z)=\frac{s_{\lambda}^{2}\left(I_{m}\right)}{s_{\lambda}\left(I_{n}\right)},
$$

where $Z$ are complex $m \times m$ matrices, and $I_{m}$ and $I_{n}$ are identity matrices of sizes $m \times m$ and $n \times n$, respectively. The first identity holds for any non-negative integer $n$ and any partition $\lambda$ such that $l\left(\lambda^{\prime}\right) \leq n$. The second one holds for any integer $n \geq 2 m$ and any $\lambda$. Since the above identities become trivial (both sides vanish) for partitions of length $>m$ we drop the restriction $l(\lambda) \leq m$.

These two identities lead to several useful matrix integrals.

Let $M$ be an $m \times m$ matrix. Then, for any non-negative integer $n$,

$$
\int_{Z Z^{*} \geq 0} s_{\lambda}\left(M Z Z^{*}\right) d \hat{\mu}_{n}(Z)=\frac{s_{\lambda}(M) s_{\lambda}\left(I_{m}\right)}{s_{\lambda^{\prime}}\left(I_{n}\right)}
$$


provided $l\left(\lambda^{\prime}\right) \leq n$, and if $n \geq 2 m$ then

$$
\int_{Z Z^{*} \leq I_{m}} s_{\lambda}\left(M Z Z^{*}\right) d \hat{\nu}_{n}(Z)=\frac{s_{\lambda}(M) s_{\lambda}\left(I_{m}\right)}{s_{\lambda}\left(I_{n}\right)}
$$

for any $\lambda$. These two integrals follow from (3.27) and (3.8) and the unitary invariance of $d \hat{\mu}_{n}(Z)$ and $d \hat{\nu}_{n}(Z)$.

If $L$ and $M$ are two $m \times m$ matrices then for any non-negative integer $n$

$$
\int_{Z Z^{*} \geq 0} s_{\lambda}(L Z) \overline{s_{\mu}(M Z)} d \hat{\mu}_{n}(Z)=\delta_{\lambda, \mu} \frac{s_{\lambda}\left(L M^{*}\right)}{s_{\lambda^{\prime}}\left(I_{n}\right)},
$$

provided $l\left(\lambda^{\prime}\right) \leq n$ and $l\left(\mu^{\prime}\right) \leq n$, and if $n \geq 2 m$ then

$$
\int_{Z Z^{*} \leq I_{m}} s_{\lambda}(L Z) \overline{s_{\mu}(M Z)} d \hat{\nu}_{n}(Z)=\delta_{\lambda, \mu} \frac{s_{\lambda}\left(L M^{*}\right)}{s_{\lambda}\left(I_{n}\right)},
$$

These orthogonality relations follow from (3.7) and (3.28) - (3.29), and, in turn, lead to Berezin-Hua integrals [30, 7]

$$
\begin{aligned}
\int_{Z Z^{*} \geq 0} \operatorname{det}^{n}\left(I_{m}+L Z\right) \operatorname{det}^{n}\left(I_{m}+M Z\right)^{*} d \hat{\mu}_{n}(Z) & =\operatorname{det}^{n}\left(I_{m}+L M^{*}\right) \\
\int_{Z Z^{*} \leq I_{m}} \frac{d \hat{\nu}_{n}(Z)}{\operatorname{det}^{n}\left(I_{m}-L Z\right) \operatorname{det}^{n}\left(I_{m}-M Z\right)^{*}} & =\frac{1}{\operatorname{det}^{n}\left(I_{m}-L M^{*}\right)}, n \geq 2 m .
\end{aligned}
$$

One only has to recall the Cauchy identites (3.13) and (3.14).

If $P$ and $Q$ are two $n \times m$ matrices and $n \geq 2 m$ then it follows from (3.7) and (3.31) that

$$
\int_{U(n)} s_{\lambda}\left(P Q^{*} U\right) \overline{s_{\mu}\left(P Q^{*} U\right)} d U=\int_{Z Z^{*} \leq I_{m}} s_{\lambda}\left(P^{*} P Z\right) \overline{s_{\mu}\left(Q^{*} Q Z\right)} d \hat{\nu}_{n}(Z)
$$

for any $\lambda$ and $\mu$. Identity (3.32) implies that

$$
\int_{U(n)} e^{\operatorname{tr}\left(P Q^{*} U+U^{*} Q P^{*}\right)} d U=\int_{Z Z^{*} \leq I_{m}} e^{\operatorname{tr}\left(P^{*} P Z+Z^{*} Q^{*} Q\right)} d \hat{\nu}_{n}(Z) .
$$

The duality relation (3.33) is a particular case of Zirnbauer's color-flavor transformation [48. It can be easily obtained from (3.32) by making use of the expansion

$$
e^{\operatorname{tr} A}=\sum_{\lambda} c_{\lambda} s_{\lambda}(A)
$$

In fact, (3.33) extends to any series $g(A)=\sum_{\lambda} c_{\lambda} s_{\lambda}(A)$,

$$
\int_{U(n)}\left|g\left(P Q^{*} U\right)\right|^{2} d U=\int_{Z Z^{*} \leq I_{m}} g\left(P^{*} P Z\right) \overline{g\left(Q^{*} Q Z\right)} d \hat{\nu}_{n}(Z) .
$$


It follows from (3.34) and (3.7) that the integral over the unitary group on the lefthand side in (3.33) is a function of $Q^{*} Q P^{*} P$. This function can be evaluated explicitly in terms of the eigenvalues of $Q^{*} Q P^{*} P$. We would like to demonstrate this in a slightly more general setting.

For square matrices $A$ and $B$ of size $n \times n$ define

$$
F_{n}\left(A B^{*}\right)=\int_{U(n)} e^{\operatorname{tr}\left(A U+U^{*} B^{*}\right)} d U
$$

If the eigenvalues $z_{1}^{2}, \ldots, z_{n}^{2}$ of the matrix $A B^{*}$ are all distinct then [4]

$$
F_{n}\left(A B^{*}\right)=\frac{\text { Const. }}{\Delta\left(z_{1}^{2}, \ldots, z_{n}^{2}\right)} \times \operatorname{det}\left(z_{i}^{j-1} I_{j-1}\left(z_{i}\right)\right)_{i, j=1}^{n}
$$

where $I_{k}$ is the modified Bessel function,

$$
I_{k}(z)=\sum_{j=0}^{\infty} \frac{\left(\frac{z}{2}\right)^{2 j+k}}{j !(j+k) !}
$$

For our purposes, we want to know $F_{n}\left(A B^{*}\right)$ for matrices $A B^{*}$ of low rank, e.g. when $A B^{*}$ is rank one.

Lemma 5 Suppose that $A B^{*}$ has $m$ distinct non-zero eigenvalues $z_{1}^{2}, \ldots, z_{m}^{2}$ and $2 m \leq n$. Then

$$
F_{n}\left(A B^{*}\right)=\left(\prod_{j=1}^{m} \frac{(n-j) !}{(n-m-j) !}\right) \int_{0}^{1} \ldots \int_{0}^{1} \frac{\operatorname{det}\left(g\left(t_{i} z_{j}^{2}\right)\right)_{i, j=1}^{m}}{\Delta\left(z_{1}^{2}, \ldots, z_{m}^{2}\right)} \prod_{i=1}^{m} t_{i}^{m-i}\left(1-t_{i}\right)^{n-2 m} d t_{i}
$$

where $g(x)=I_{0}(2 \sqrt{x})$. In particular, if $A B^{*}$ is rank one and $z^{2}$ is its non-zero eigenvalue then

$$
F_{n}\left(A B^{*}\right)=(n-1) \int_{0}^{1} I_{0}\left(2 \sqrt{t z^{2}}\right)(1-t)^{n-2} d t
$$

Proof. It follows from (3.34) and (3.7) that

$$
F_{n}\left(A B^{*}\right)=\sum_{\lambda} \frac{c_{\lambda}^{2}}{s_{\lambda}\left(1_{n}\right)} s_{\lambda}\left(A B^{*}\right)
$$

The coefficients $c_{\lambda}$ are given by

$$
c_{\lambda}=\operatorname{det}\left(\frac{1}{\left(\lambda_{j}-j+i\right) !}\right)_{i, j=1}^{m}=s_{\lambda}\left(1_{m}\right) \prod_{j=1}^{m} \frac{(m-j) !}{\left(m+\lambda_{j}-j\right) !}
$$


see, e.g., 4] and references therein, and

$$
F_{n}\left(A B^{*}\right)=\left(\prod_{j=0}^{m-1} j !^{2}\right) \sum_{\lambda} \frac{s_{\lambda}^{2}\left(1_{m}\right)}{s_{\lambda}\left(1_{n}\right)} \frac{s_{\lambda}\left(A B^{*}\right)}{f_{1} !^{2} \cdot \ldots \cdot f_{m} !^{2}}
$$

where as before $f_{j}=m+\lambda_{j}-j$. Note that the summation is over all partitions $\lambda$ of length $\leq m$, or, equivalently, over all $f_{1}>f_{2}>\ldots>f_{m} \geq 0$. It follows now from (3.21) -3.22 that

$$
F_{n}\left(A B^{*}\right)=\left(\prod_{j=1}^{m} \frac{(n-j) !}{(n-m-j) !}\right) \int_{0}^{1} \ldots \int_{0}^{1} \frac{g\left(t_{1}, \ldots t_{m}\right)}{\Delta\left(z_{1}^{2}, \ldots, z_{m}^{2}\right)} \prod_{i=1}^{m} t_{i}^{m-i}\left(1-t_{i}\right)^{n-2 m} d t_{i} .
$$

where

$$
g\left(t_{1}, \ldots t_{m}\right)=\sum_{f_{1}>f_{2}>\ldots>f_{m} \geq 0} \frac{\operatorname{det}\left(t_{i}^{f_{j}}\right)_{i, j=1}^{m} \operatorname{det}\left(z_{i}^{2 f_{j}}\right)_{i, j=1}^{m}}{f_{1} !^{2} \ldots f_{m} !^{2}} .
$$

To complete the proof, recall the following generalization of the Cauchy-Binet formula, see e.g. [30] p. 22. If $g(x)=\sum_{f \geq 0} \gamma_{f} x^{f}$ is an analytic function in the complex $x$-plane then

$$
\operatorname{det}\left(g\left(t_{i} x_{j}\right)\right)_{i, j=1}^{m}=\sum_{f_{1}>f_{2}>\ldots>f_{m} \geq 0} \gamma_{f_{1}} \ldots \gamma_{f_{m}} \operatorname{det}\left(t_{i}^{f_{j}}\right)_{i, j=1}^{m} \operatorname{det}\left(x_{i}^{f_{j}}\right)_{i, j=1}^{m} .
$$

By making use of this formula,

$$
g\left(t_{1}, \ldots, t_{m}\right)=\operatorname{det}\left(I_{0}\left(2 \sqrt{t_{i} z_{j}^{2}}\right)\right)_{i, j=1}^{m},
$$

and Lemma follows.

\section{Proof of Theorem 1}

After all the preparatory work of the previous section, Theorem 1 becomes almost evident.

We first prove (2.9) - (2.10) for $C=D=I$. With Lemma 3 in hand, this becomes a routine calculation. Expanding powers of determinants in the Schur functions as in (3.13)

- (3.14) and integrating over the unitary group with the help of (3.7), one gets

$$
\int_{U(n)} \operatorname{det}^{m}\left[(I+A U)(I+B U)^{*}\right] d U=\sum_{\lambda} \frac{s_{\lambda}^{2}\left(1_{m}\right)}{s_{\lambda^{\prime}}\left(1_{n}\right)} s_{\lambda^{\prime}}\left(A B^{*}\right)
$$

and

$$
\int_{U(n)} \frac{d U}{\operatorname{det}^{m}\left[(I-A U)(I-B U)^{*}\right]}=\sum_{\lambda} \frac{s_{\lambda}^{2}\left(1_{m}\right)}{s_{\lambda}\left(1_{n}\right)} s_{\lambda}\left(A B^{*}\right)
$$


The sum in (4.1) is finite and the sum in (4.2) is absolutely converging for any $A A^{*}<I$ and $B B^{*}<I$. Now, by making use of (3.18) and (3.19), and then (3.7) again, one arrives at

$$
\int_{U(n)} \operatorname{det}^{m}\left[(I+A U)(I+B U)^{*}\right] d U=\int_{0}^{\infty} \ldots \int_{0}^{\infty} \prod_{j=1}^{m} \operatorname{det}\left(I+t_{j} A B^{*}\right) d \mu_{n}\left(t_{1}, \ldots, t_{m}\right)
$$

and

$$
\int_{U(n)} \frac{d U}{\operatorname{det}^{m}\left[(I-A U)(I-B U)^{*}\right]}=\int_{0}^{1} \ldots \int_{0}^{1} \frac{d \nu_{n}\left(t_{1}, \ldots, t_{m}\right)}{\prod_{j=1}^{m} \operatorname{det}\left(I-t_{j} A B^{*}\right)} .
$$

Extending (4.3) and (4.4) to the generality of (2.9) and (2.10) is straightforward. If $C$ and $D$ are not degenerate, then

$$
\int_{U(n)} \operatorname{det}^{m}\left[(A U+C)(B U+D)^{*}\right] d U=\operatorname{det}^{m}\left(C D^{*}\right) \int \operatorname{det}_{U(n)}^{m}\left[\left(I+C^{-1} A U\right)\left(I+D^{-1} B U\right)^{*}\right] d U
$$

and (2.9) follows from (4.3). The assumption that $C$ and $D$ are not degenerate can be removed by the continuity argument.

\section{Regularization of the inverse determinant}

In this section we employ another approach to the problem of evaluating of negative moments of spectral determinants which is to write the determinants as Gaussian integrals and then perform the integration over the unitary group with the help of the color-flavor transformation. This approach is not new. It was pioneered by Zirnbauer in the context of unitary random matrix ensembles. The new element here is that we apply it in the general context of complex matrices.

We shall write the spectral determinant $\operatorname{det}\left[(I-A U)(I-A U)^{*}\right]$ of $n \times n$ matrices as $2 n \times 2 n$ block determinant

$$
\operatorname{det}\left[(I-A U)(I-A U)^{*}\right]=\operatorname{det}\left[\left(U^{*}-A\right)\left(U^{*}-A\right)^{*}\right]=\left|\begin{array}{cc}
0 & i\left(U^{*}-A\right) \\
i\left(U^{*}-A\right)^{*} & 0
\end{array}\right|
$$

and more generally

$$
\operatorname{det}\left[\varepsilon^{2} I+(I-A U)(I-A U)^{*}\right]=\left|\begin{array}{cc}
\varepsilon I & i\left(U^{*}-A\right) \\
i\left(U^{*}-A\right)^{*} & \varepsilon I
\end{array}\right|
$$


Proposition 6 Suppose that $\operatorname{Re} \lambda_{j}>0, j=1,2$. Then for any complex $n \times n$ matrix $\Omega$ we have

$$
\left|\begin{array}{cc}
\lambda_{1} I & i \Omega \\
i \Omega^{*} & \lambda_{2} I
\end{array}\right|^{-1}=\frac{1}{\pi^{n}} \int_{\mathbb{C}^{n}} d^{2} \boldsymbol{v} \int_{\mathbb{C}^{n}} d^{2} \boldsymbol{w} \exp \left\{-\left[\lambda_{1} \boldsymbol{v}^{*} \boldsymbol{v}+\lambda_{2} \boldsymbol{w}^{*} \boldsymbol{w}+i\left(\boldsymbol{w}^{*} \Omega^{*} \boldsymbol{v}+\boldsymbol{v}^{*} \Omega \boldsymbol{w}\right)\right]\right\} .
$$

The integral on the right-hand side converges absolutely.

Remark. In this section, we shall use letters in bold face to represent column vectors in $\mathbb{C}^{n}$. The symbol $d^{2} \boldsymbol{v}$ will denote the volume element of $\boldsymbol{v}$ in $\mathbb{C}^{n}$,

$$
d^{2} \boldsymbol{v}=\prod_{j=1}^{n} d^{2} \eta_{j}=\prod_{j=1}^{n} d \operatorname{Re} v_{j} d \operatorname{Im} v_{j}
$$

Proof. Note that

$$
\lambda_{1} \boldsymbol{v}^{*} \boldsymbol{v}+\lambda_{2} \boldsymbol{w}^{*} \boldsymbol{w}+i\left(\boldsymbol{w}^{*} \Omega^{*} \boldsymbol{v}+\boldsymbol{v}^{*} \Omega \boldsymbol{w}\right)=\left(\boldsymbol{v}^{*}, \boldsymbol{w}^{*}\right)\left(\begin{array}{cc}
\lambda_{1} I & i \Omega \\
i \Omega^{*} & \lambda_{2} I
\end{array}\right)\left(\begin{array}{c}
\boldsymbol{v} \\
\boldsymbol{w}
\end{array}\right) .
$$

In view of the singular value decomposition $\Omega=U^{*} \omega V$,

$$
\left(\begin{array}{cc}
\lambda_{1} I & i \Omega \\
i \Omega^{*} & \lambda_{2} I
\end{array}\right)=\left(\begin{array}{cc}
U^{*} & 0 \\
0 & V^{*}
\end{array}\right)\left(\begin{array}{cc}
\lambda_{1} I & i \omega \\
i \omega & \lambda_{2} I
\end{array}\right)\left(\begin{array}{cc}
U & 0 \\
0 & V
\end{array}\right)
$$

where $\omega$ is diagonal matrix of singular values of $\Omega, \omega=\operatorname{diag}\left(\omega_{1}, \ldots, \omega_{n}\right)$, and $U$ and $V$ are unitary matrices. Introducing $\boldsymbol{f}=U \boldsymbol{v}$ and $\boldsymbol{g}=V \boldsymbol{w}$,

$$
\begin{aligned}
\left(\boldsymbol{v}^{*}, \boldsymbol{w}^{*}\right)\left(\begin{array}{cc}
\lambda_{1} I & i \Omega \\
i \Omega^{*} & \lambda_{2} I
\end{array}\right)\left(\begin{array}{c}
\boldsymbol{v} \\
\boldsymbol{w}
\end{array}\right) & =\left(\boldsymbol{f}^{*}, \boldsymbol{g}^{*}\right)\left(\begin{array}{cc}
\lambda_{1} I & i \omega \\
i \omega & \lambda_{2} I
\end{array}\right)\left(\begin{array}{l}
\boldsymbol{f} \\
\boldsymbol{g}
\end{array}\right) \\
& =\sum_{j=1}^{n}\left(\bar{f}_{j}, \bar{g}_{j}\right)\left(\begin{array}{cc}
\lambda_{1} & i \omega_{j} \\
i \omega_{j} & \lambda_{2}
\end{array}\right)\left(\begin{array}{l}
f_{j} \\
g_{j}
\end{array}\right) .
\end{aligned}
$$

Since $U$ and $V$ are unitary, $d^{2} \boldsymbol{v}=d^{2} \boldsymbol{f}$ and $d^{2} \boldsymbol{w}=d^{2} \boldsymbol{g}$. Changing the variables of integration in (5.1) from $\boldsymbol{v}$ and $\boldsymbol{w}$ to $\boldsymbol{f}$ and $\boldsymbol{g}$ breaks this $2 n$-fold integral into the product of the 2-fold integrals

$$
\frac{1}{\pi} \int_{\mathbb{C}^{2}} \exp \left[-\lambda_{1}\left|f_{j}\right|^{2}-\lambda_{2}\left|g_{j}\right|^{2}-i \omega_{j}\left(f_{j} \bar{g}_{j}+\bar{f}_{j} g_{j}\right)\right] d^{2} f_{j} d^{2} g_{j}=\left(\lambda_{1} \lambda_{2}+\omega_{j}^{2}\right)^{-1} .
$$

Thus, the integral on the right-hand side in (5.1) equals $\prod_{j=1}\left(\lambda_{1} \lambda_{2}+\omega_{j}^{2}\right)^{-1}$ which is obviously same as the determinant on the left-hand side.

Proof of Theorem Q Obviously, without loss of generality we can put $z=1$. Let

$$
R_{\varepsilon}\left(A, A^{*}\right)=\int_{U(n)} \frac{d U}{\operatorname{det}\left[\varepsilon^{2} I+(I-A U)(I-A U)^{*}\right]}, \quad \varepsilon>0 .
$$


The integral on the right-hand side converges for any $n \times n$ matrix $A$.

It follows from Proposition 6 that

$$
R_{\varepsilon}\left(A, A^{*}\right)=\frac{1}{\pi^{n}} \int_{\mathbb{C}^{n}} d^{2} \boldsymbol{v} \int_{\mathbb{C}^{n}} d^{2} \boldsymbol{w} e^{-\left[\varepsilon\left(\boldsymbol{v}^{*} \boldsymbol{v}+\boldsymbol{w}^{*} \boldsymbol{w}\right)-i\left(\boldsymbol{w}^{*} A^{*} \boldsymbol{v}+\boldsymbol{v}^{*} A \boldsymbol{w}\right)\right]} f_{n}\left(\boldsymbol{v}^{*} \boldsymbol{v} \boldsymbol{w}^{*} \boldsymbol{w}\right),
$$

where, cf. (3.35),

$$
f_{n}\left(\boldsymbol{v}^{*} \boldsymbol{v} \boldsymbol{w}^{*} \boldsymbol{w}\right)=\int_{U(n)} e^{i\left(\boldsymbol{w}^{*} U \boldsymbol{v}+\boldsymbol{v}^{*} U^{*} \boldsymbol{w}\right)} d U=\int_{U(n)} e^{i \operatorname{tr}\left(\boldsymbol{v} \boldsymbol{w}^{*} U+U^{*} \boldsymbol{w} \boldsymbol{v}^{*}\right)} d U
$$

By Lemma 5 ,

$$
f_{n}\left(\boldsymbol{v}^{*} \boldsymbol{v} \boldsymbol{w}^{*} \boldsymbol{w}\right)=\int_{0}^{1} J_{0}\left(2 \sqrt{t \boldsymbol{v}^{*} \boldsymbol{v} \boldsymbol{w}^{*} \boldsymbol{w}}\right) d \sigma_{n}(t)
$$

where

$$
d \sigma_{n}(t)=(n-1)(1-t)^{n-2} d t
$$

and $J_{0}$ is the Bessel function

$$
J_{0}(z)=\sum_{j=0}^{\infty} \frac{\left(\frac{i z}{2}\right)^{2 j}}{j !^{2}}=I_{0}(i z)
$$

We have $\left|f_{n}\left(\boldsymbol{v}^{*} \boldsymbol{v} \boldsymbol{w}^{*} \boldsymbol{w}\right)\right| \leq 1$ for all $\boldsymbol{v}$ and $\boldsymbol{w}$. This is because $\left|J_{0}(z)\right| \leq 1$ for all $z$. Therefore we can interchange the order of integrations on replacing $f_{n}$ in (5.3) by the integral of (5.4). This yields

$$
R_{\varepsilon}\left(A, A^{*}\right)=\int_{0}^{1} d \sigma_{n}(t) \frac{1}{\pi^{n}} \int_{\mathbb{C}^{n}} d^{2} \boldsymbol{v} \int_{\mathbb{C}^{n}} d^{2} \boldsymbol{w} e^{-\varepsilon\left(\boldsymbol{v}^{*} \boldsymbol{v}+\boldsymbol{w}^{*} \boldsymbol{w}\right)+i\left(\boldsymbol{w}^{*} A^{*} \boldsymbol{v}+\boldsymbol{v}^{*} A \boldsymbol{w}\right)} J_{0}\left(2 \sqrt{t \boldsymbol{v}^{*} \boldsymbol{v} \boldsymbol{w}^{*} \boldsymbol{w}}\right) .
$$

In order to perform the integration in variables $\boldsymbol{v}$ and $\boldsymbol{w}$ we shall make use of the integral representation

$$
J_{0}(2 \sqrt{p q})=\frac{1}{2 \pi i} \int_{-\infty}^{+\infty} \frac{d x}{x} e^{i\left(p x+\frac{q}{x}\right)}
$$

which holds any $p>0$ and $q>0$ and is a particular case of equation 3.871.1 in [32. The integral in (5.6) converges because of the oscillations of the exponential function, however the convergence is not absolute.

We have

$$
J_{0}\left(2 \sqrt{t \boldsymbol{v}^{*} \boldsymbol{v} \boldsymbol{w}^{*} \boldsymbol{w}}\right)=\frac{1}{2 \pi i} \int_{-\infty}^{+\infty} \frac{d x}{x} e^{i \sqrt{t}\left(x \boldsymbol{v}^{*} \boldsymbol{v}+\frac{\boldsymbol{w}^{*} \boldsymbol{w}}{x}\right)} .
$$

Note that by Proposition [6]

$$
\frac{1}{\pi^{n}} \int_{\mathbb{C}^{n}} d^{2} \boldsymbol{v} \int_{\mathbb{C}^{n}} d^{2} \boldsymbol{w} e^{-\varepsilon\left(\boldsymbol{v}^{*} \boldsymbol{v}+\boldsymbol{w}^{*} \boldsymbol{w}\right)+i\left(\boldsymbol{w}^{*} A^{*} \boldsymbol{v}+\boldsymbol{v}^{*} A \boldsymbol{w}\right)} e^{i \sqrt{t}\left(x \boldsymbol{v}^{*} \boldsymbol{v}+\frac{\boldsymbol{w}^{*} \boldsymbol{w}}{x}\right)}=\left|\begin{array}{cc}
(\varepsilon-i \sqrt{t} x) I & -A \\
-A^{*} & \left(\varepsilon-i \frac{\sqrt{t}}{x}\right) I
\end{array}\right|^{-1} .
$$


Therefore, on replacing $J_{0}$ in (5.5) by the integral of (15.7) and changing the order of integrations one arrives at

$$
R_{\varepsilon}\left(A, A^{*}\right)=\int_{0}^{1} d \sigma_{n}(t) \frac{1}{2 \pi i} \int_{-\infty}^{+\infty} \frac{d x}{x} \frac{1}{\operatorname{det}\left[A A^{*}+\left(\varepsilon^{2}-t\right) I-i \varepsilon \sqrt{t}\left(x+\frac{1}{x}\right) I\right]}
$$

which is the identity claimed in Theorem 2, It remains to justify reversing the order of integrations with respect to $x$ and $\boldsymbol{v}, \boldsymbol{w}$. Firstly, we will show that the integral on the right-hand side in (5.8) is well-defined.

Proposition 7 For any $\varepsilon>0$ and $n \geq 2$ the integral in (5.8) converges absolutely (and uniformly in A).

Proof. Let $a_{j}^{2}$ be the eigenvalues of $A A^{*}$ so that

$$
\frac{1}{x} \times \frac{1}{\operatorname{det}\left[A A^{*}+\left(\varepsilon^{2}-t\right) I-i \varepsilon \sqrt{t}\left(x+\frac{1}{x}\right) I\right]}=\frac{1}{x} \prod_{j=1}^{n} w\left(a_{j}, t\right)
$$

where

$$
w(a, t)=\frac{1}{a^{2}+\varepsilon^{2}-t-i \varepsilon \sqrt{t}\left(x+\frac{1}{x}\right)} .
$$

Since $|z| \geq|\operatorname{Im} z|$, we have

$$
\left|\frac{1}{x} w\left(a_{1}, t\right)\right| \leq \frac{1}{\varepsilon \sqrt{t}\left(1+x^{2}\right)} .
$$

Also, for all $0 \leq t \leq \varepsilon^{2} / 2$ we have

$$
\left|w\left(a_{j}, t\right)\right| \leq \frac{1}{\left|\varepsilon^{2}-t+a_{j}^{2}\right|} \leq \frac{2}{\varepsilon^{2}}
$$

and for all $\varepsilon^{2} / 2 \leq t \leq 1$ we have

$$
\left|w\left(a_{j}, t\right)\right| \leq \frac{1}{\left|\varepsilon \sqrt{t}\left(x+\frac{1}{x}\right)\right|} \leq \frac{1}{2 \varepsilon \sqrt{t}} \leq \frac{1}{\sqrt{2} \varepsilon^{2}} .
$$

Therefore the absolute value of the integrand in (5.8) is majorated by the function

$$
\frac{1}{\varepsilon \sqrt{t}\left(1+x^{2}\right)}\left(\frac{2}{\varepsilon^{2}}\right)^{n-1}
$$

which is obviously integrable with respect to $d \sigma_{n}(t) \times d x$.

We can now turn to justification of reversing the order of integrations in $\boldsymbol{v}, \boldsymbol{w}$ and $x$ in the integral

$$
\mathcal{I}=\int_{0}^{1} d \sigma_{n}(t) \int_{\mathbb{C}^{n}} d^{2} \boldsymbol{v} \int_{\mathbb{C}^{n}} d^{2} \boldsymbol{w} e^{-\varepsilon\left(\boldsymbol{v}^{*} \boldsymbol{v}+\boldsymbol{w}^{*} \boldsymbol{w}\right)+i\left(\boldsymbol{w}^{*} A^{*} \boldsymbol{v}+\boldsymbol{v}^{*} A \boldsymbol{w}\right)} \int_{-\infty}^{+\infty} \frac{d x}{x} e^{i \sqrt{t}\left(x \boldsymbol{v}^{*} \boldsymbol{v}+\frac{\boldsymbol{w}^{*} \boldsymbol{w}}{x}\right)}
$$


The corresponding calculation is routine but tedious. First we restrict the $x$-integration to the finite interval $\delta \leq|x| \leq 1 / \delta, \delta>0$, reverse the order of integrations, and then show that the corresponding tail integrals in are negligible in the limit $\delta \rightarrow 0$.

Let

$$
\mathcal{I}_{\delta}=\int_{0}^{1} d \sigma_{n}(t) \int_{\mathbb{C}^{n}} d^{2} \boldsymbol{v} \int_{\mathbb{C}^{n}} d^{2} \boldsymbol{w} \int_{\delta \leq|x| \leq 1 / \delta} \frac{d x}{x} e^{-\varepsilon\left(\boldsymbol{v}^{*} \boldsymbol{v}+\boldsymbol{w}^{*} \boldsymbol{w}\right)+i\left(\boldsymbol{w}^{*} A^{*} \boldsymbol{v}+\boldsymbol{v}^{*} A \boldsymbol{w}\right)} e^{i \sqrt{t}\left(x \boldsymbol{v}^{*} \boldsymbol{v}+\frac{\boldsymbol{w}^{*} \boldsymbol{w}}{x}\right)}
$$

The absolute value of the integrand is majorated by the integrable function $\frac{1}{|x|} e^{-\varepsilon\left(\boldsymbol{v}^{*} \boldsymbol{v}+\boldsymbol{w}^{*} \boldsymbol{w}\right)}$, and therefore we can reverse the order of integrations and then perform the integration in $\boldsymbol{v}, \boldsymbol{w}$. This yields

$$
\mathcal{I}_{\delta}=\int_{0}^{1} d \sigma_{n}(t) \int_{\delta \leq|x| \leq 1 / \delta} \frac{d x}{x} \frac{1}{\operatorname{det}\left[A A^{*}+\left(\varepsilon^{2}-t\right) I-i \varepsilon \sqrt{t}\left(x+\frac{1}{x}\right) I\right]} .
$$

It follows from this, in view of By Proposition [7] that

$$
\mathcal{I}_{\delta}=\int_{0}^{1} d \sigma_{n}(t) \int_{-\infty}^{+\infty} \frac{d x}{x} \frac{1}{\operatorname{det}\left[A A^{*}+\left(\varepsilon^{2}-t\right) I-i \varepsilon \sqrt{t}\left(x+\frac{1}{x}\right) I\right]}+o(1)
$$

in the limit $\delta \rightarrow 0$. It only remains to show that the tail integrals

$$
\mathcal{I}_{\delta}^{\prime}=\int_{0}^{1} d \sigma_{n}(t) \int_{\mathbb{C}^{n}} d^{2} \boldsymbol{v} \int_{\mathbb{C}^{n}} d^{2} \boldsymbol{w} e^{-\varepsilon\left(\boldsymbol{v}^{*} \boldsymbol{v}+\boldsymbol{w}^{*} \boldsymbol{w}\right)+i\left(\boldsymbol{w}^{*} A^{*} \boldsymbol{v}+\boldsymbol{v}^{*} A \boldsymbol{w}\right)} \int_{|x| \geq 1 / \delta} \frac{d x}{x} e^{i \sqrt{t}\left(x \boldsymbol{v}^{*} \boldsymbol{v}+\frac{\boldsymbol{w}^{*} \boldsymbol{w}}{x}\right)}
$$

and

$$
\mathcal{I}_{\delta}^{\prime \prime}=\int_{0}^{1} d \sigma_{n}(t) \int_{\mathbb{C}^{n}} d^{2} \boldsymbol{v} \int_{\mathbb{C}^{n}} d^{2} \boldsymbol{w} e^{-\varepsilon\left(\boldsymbol{v}^{*} \boldsymbol{v}+\boldsymbol{w}^{*} \boldsymbol{w}\right)+i\left(\boldsymbol{w}^{*} A^{*} \boldsymbol{v}+\boldsymbol{v}^{*} A \boldsymbol{w}\right)} \int_{|x| \leq \delta} \frac{d x}{x} e^{i \sqrt{t}\left(x \boldsymbol{v}^{*} \boldsymbol{v}+\frac{\boldsymbol{w}^{*} \boldsymbol{w}}{x}\right)}
$$

vanish in the limit $\delta \rightarrow 0$.

For real $r, p$ and $q$ define

$$
g_{L}(r, p, q)=\int_{L}^{+\infty} \frac{d x}{x} e^{i r\left(p x+\frac{q}{x}\right)}=\int_{0}^{1 / L} \frac{d x}{x} e^{i r\left(q x+\frac{p}{x}\right)} .
$$

By integrating by parts,

$$
g_{L}(t ; p, q)=-\frac{1}{i p r L} e^{i r\left(p L+\frac{q}{L}\right)}+\frac{1}{i p r} \int_{L}^{+\infty} \frac{e^{i r\left(p x+\frac{q}{x}\right)}}{x^{2}} d x+\frac{p}{q} \int_{L}^{+\infty} \frac{e^{i r\left(p x+\frac{q}{x}\right)}}{x^{3}} d x
$$

and, therefore, for $L>0$ we have

$$
\left|g_{L}(r, p, q)\right| \leq \frac{2}{|p||r| L}+\frac{|q|}{2|p| L^{2}}
$$


Obviously,

$$
\int_{|x| \geq 1 / \delta} \frac{d x}{x} e^{i \sqrt{t}\left(x \boldsymbol{v}^{*} \boldsymbol{v}+\frac{\boldsymbol{w}^{*} \boldsymbol{w}}{x}\right)}=g_{\frac{1}{\delta}}\left(\sqrt{t}, \boldsymbol{v}^{*} \boldsymbol{v}, \boldsymbol{w}^{*} \boldsymbol{w}\right)-g_{\frac{1}{\delta}}\left(-\sqrt{t}, \boldsymbol{v}^{*} \boldsymbol{v}, \boldsymbol{w}^{*} \boldsymbol{w}\right),
$$

and, by (5.11),

$$
\left|\int_{|x| \leq \delta} \frac{d x}{x} e^{i \sqrt{t}\left(x \boldsymbol{v}^{*} \boldsymbol{v}+\frac{\boldsymbol{w}^{*} \boldsymbol{w}}{x}\right)}\right| \leq \frac{4 \delta}{\boldsymbol{v}^{*} \boldsymbol{v} \sqrt{t}}+\frac{\boldsymbol{w}^{*} \boldsymbol{w} \delta^{2}}{\boldsymbol{v}^{*} \boldsymbol{v}}
$$

Therefore

$$
\left|\mathcal{I}_{\delta}^{\prime}\right| \leq \int_{0}^{1} d \sigma_{n}(t) \int_{\mathbb{C}^{n}} d^{2} \boldsymbol{v} \int_{\mathbb{C}^{n}} d^{2} \boldsymbol{w} e^{-\varepsilon\left(\boldsymbol{v}^{*} \boldsymbol{v}+\boldsymbol{w}^{*} \boldsymbol{w}\right)}\left(\frac{4 \delta}{\boldsymbol{v}^{*} \boldsymbol{v} \sqrt{t}}+\frac{\boldsymbol{w}^{*} \boldsymbol{w} \delta^{2}}{\boldsymbol{v}^{*} \boldsymbol{v}}\right) .
$$

As the function $\frac{1}{\boldsymbol{v}^{*} \boldsymbol{v}}$ is locally integrable with respect to $d^{2} \boldsymbol{v}$ for $n \geq 2$, we conclude that

$$
\mathcal{I}_{\delta}^{\prime}=O(\delta) \quad \text { when } \delta \rightarrow 0
$$

Similarly

$$
\int_{|x| \leq \delta} \frac{d x}{x} e^{i \sqrt{t}\left(x \boldsymbol{v}^{*} \boldsymbol{v}+\frac{\boldsymbol{w}^{*} \boldsymbol{w}}{x}\right)}=g_{\frac{1}{\delta}}\left(\sqrt{t}, \boldsymbol{w}^{*} \boldsymbol{w}, \boldsymbol{v}^{*} \boldsymbol{v}\right)-g_{\frac{1}{\delta}}\left(-\sqrt{t}, \boldsymbol{w}^{*} \boldsymbol{w}, \boldsymbol{v}^{*} \boldsymbol{v}\right),
$$

and repeating the above argument one obtains that

$$
\mathcal{I}_{\delta}^{\prime \prime}=O(\delta) \quad \text { when } \delta \rightarrow 0
$$

so that both $\mathcal{I}_{\delta}^{\prime}$ and $\mathcal{I}_{\delta}^{\prime \prime}$ vanish in the limit $\delta \rightarrow 0$. This completes our proof of the first part of Theorem 2 .

It is worth mentioning another formula for the regularized average of the inverse spectral determinant,

$$
R_{\varepsilon}\left(A, A^{*}\right)=(n-1) \int_{|z|^{2} \leq 1} \frac{\left(1-|z|^{2}\right)^{n-2} d^{2} z}{\operatorname{det}\left[\varepsilon^{2} I+(1-\bar{z}) I+(1-z) A A^{*}\right]},
$$

which is almost an immediate corollary of (3.33) and the representation

$$
\begin{aligned}
\frac{1}{\operatorname{det}\left[\varepsilon^{2} I_{n}+\left(I_{n}-A U\right)\left(I_{n}-A U\right)^{*}\right]} & =\frac{1}{\pi^{n}} \int_{\mathbb{C}^{n}} e^{-\boldsymbol{v}^{*}\left[\varepsilon^{2} I+(I-A U)(I-A U)^{*}\right] \boldsymbol{v}} d^{2} \boldsymbol{v} \\
& =\frac{1}{\pi^{n}} \int_{\mathbb{C}^{n}} e^{-\left[\boldsymbol{v}^{*}\left(1+\varepsilon^{2}\right) \boldsymbol{v}+\boldsymbol{v}^{*} A A^{*} \boldsymbol{v}\right]} e^{\operatorname{tr}\left(\boldsymbol{v} \boldsymbol{v}^{*} A U+U^{*} A^{*} \boldsymbol{v} \boldsymbol{v}^{*}\right)} d^{2} \boldsymbol{v}
\end{aligned}
$$

This formula, however, does not seem to be easy to handle in the limit $\varepsilon \rightarrow 0$.

We now turn to the integral in (5.8) and evaluate it in the limit $\varepsilon \rightarrow 0$ under the assumption that $A A^{*}$ has no repeated eigenvalues. The limit in (2.15) follows immediately from the asymptotic relation (5.20) which is the end-product of our calculation.

The following identities, which can be obtained from the Lagrange interpolation formula, see, e.g., [40], will be useful for our purposes. 
Proposition 8 Suppose that $x_{1}, \ldots, x_{n}$ are pairwise distinct. Then

$$
\prod_{j=1}^{n} \frac{1}{x_{j}-t}=\sum_{j=1}^{n} \frac{1}{x_{j}-t} \prod_{k \neq j} \frac{1}{x_{k}-x_{j}}
$$

and, for non-negative integer $r$,

$$
\sum_{j=1}^{n} x_{j}^{r} \prod_{k \neq j} \frac{1}{x_{k}-x_{j}}= \begin{cases}0 & \text { if } r \leq n-2, \\ h_{r-n+1}\left(x_{1}, \ldots, x_{n}\right) & \text { if } r \geq n-1\end{cases}
$$

where the $h_{r}, r=0,1,2, \ldots$, are the complete symmetric functions.

It follows from (5.13) that

$$
\frac{1}{\operatorname{det}\left[A A^{*}+\left(\varepsilon^{2}-t\right) I-i \varepsilon \sqrt{t}\left(x+\frac{1}{x}\right) I\right]}=\sum_{j=1}^{n} w\left(a_{j}, t, x\right) \prod_{k \neq j} \frac{1}{a_{k}^{2}-a_{j}^{2}},
$$

where $a_{1}, \ldots, a_{n}$ are the eigenvalues of $A A^{*}$ and

$$
w(a, t, x)=\frac{1}{a^{2}+\varepsilon^{2}-t-i \varepsilon \sqrt{t}\left(x+\frac{1}{x}\right)} .
$$

By the calculus of residues,

$$
\frac{1}{2 \pi i} \int_{-\infty}^{+\infty} \frac{d x}{x} w(a, t, x)=\frac{1}{\sqrt{\left(a^{2}-t-\varepsilon^{2}\right)^{2}+4 \varepsilon^{2} a^{2}}}
$$

and putting (5.15) and (5.16) into (5.8) we arrive at the following expression of $R_{\varepsilon}\left(A, A^{*}\right)$ in terms of the eigenvalues of $A A^{*}$ :

$$
R_{\varepsilon}\left(A, A^{*}\right)=\sum_{j=1}^{n} F_{\varepsilon}\left(a_{j}\right) \prod_{k \neq j} \frac{1}{a_{k}^{2}-a_{j}^{2}}
$$

where

$$
F_{\varepsilon}(a)=\int_{0}^{1} \frac{d \sigma_{n}(t)}{\sqrt{\left(a^{2}-t-\varepsilon^{2}\right)^{2}+4 \varepsilon^{2} a^{2}}}, \quad d \sigma_{n}(t)=(n-1)(1-t)^{n-2} d t .
$$

This formula is convenient for finding $R_{\varepsilon}\left(A, A^{*}\right)$ in the limit $\varepsilon \rightarrow 0$.

If $A A^{*}>I$, by letting $\epsilon \rightarrow 0$ in (5.17) and recalling (5.13) we immediately obtain

$$
\lim _{\varepsilon \rightarrow 0} R_{\varepsilon}\left(A, A^{*}\right)=\int_{0}^{1} \frac{d \sigma_{n}(t)}{\operatorname{det}\left(A A^{*}-t I\right)}
$$

thus reproducing the corresponding formula of Theorem 1 part (ii). If $A A^{*}<I$ or if $A A^{*}$ has eigenvalues on each side of $a^{2}=1$, evaluation of the right-hand side in (5.17) in the limit $\varepsilon \rightarrow 0$ requires some work. 
The integral in (5.18) is standard. There are different methods available to evaluate it. None seems to give an explicit expression for all parameter values. However, we are only interested in $\varepsilon \rightarrow 0$, and in this regime

$$
F_{\varepsilon}(a)=(n-1)\left(1-a^{2}\right)^{n-2}\left[\gamma_{n-2} \operatorname{sgn}\left(a^{2}-1\right)+L_{0}(\varepsilon, a)\right]+q_{n-2}\left(a^{2}\right)+O(\varepsilon),
$$

where

$$
L_{0}(\varepsilon, a)= \begin{cases}\ln \frac{1-a^{2}}{\varepsilon^{2}} & \text { if } a^{2}<1, \\ \ln \frac{a^{2}}{a^{2}-1} & \text { if } a^{2}>1 \\ \ln \frac{2}{\varepsilon} & \text { if } a^{2}=1\end{cases}
$$

$\gamma_{n-2}$ is the partial sum of the harmonic series,

$$
\gamma_{n-2}=\sum_{j=1}^{n-2} \frac{1}{j}
$$

$\operatorname{sgn}$ is the sign function, $\operatorname{sgn}(x)$ takes value 1 if $x>0,-1$ if $x<0$ and 0 if $x=0$, and $q_{n-2}\left(a^{2}\right)$ is a polynomial of degree $n-2$ in $a^{2}$ with coefficients which do not depend on $\varepsilon$. Details of derivation of (5.19) are given in Appendix $\mathrm{A}$.

Let us now put (5.19) into (5.17). In view of (5.14) the polynomial $q_{n-2}$ gives no contribution. After rearranging the remaining terms we obtain,

$$
R_{\varepsilon}\left(A, A^{*}\right)=\alpha\left(A A^{*}\right) \ln \frac{1}{\varepsilon^{2}}+\beta\left(A A^{*}\right)+O(\varepsilon),
$$

with the coefficients $\alpha$ and $\beta$ given by

$$
\begin{aligned}
& \alpha\left(A A^{*}\right)=(n-1) \sum_{j=1}^{n}\left(1-a_{j}^{2}\right)^{n-2} \theta\left(1-a_{j}^{2}\right) \prod_{k \neq j} \frac{1}{a_{k}^{2}-a_{j}^{2}} \\
& \beta\left(A A^{*}\right)=(n-1) \sum_{j=1}^{n}\left(1-a_{j}^{2}\right)^{n-2} \psi\left(a_{j}^{2}\right) \prod_{k \neq j} \frac{1}{a_{k}^{2}-a_{j}^{2}}
\end{aligned}
$$

where $\theta$ is Heaviside's step function,

$$
\theta(x)= \begin{cases}1 & \text { if } x>1 \\ \frac{1}{2} & \text { if } x=1 \\ 0 & \text { if } x<1\end{cases}
$$

and

$$
\psi\left(a^{2}\right)= \begin{cases}\gamma_{n-2}+\ln a^{2}-\ln \left(a^{2}-1\right) & \text { if } a^{2}>1 \\ -\gamma_{n-2}+\ln \left(1-a^{2}\right) & \text { if } a^{2}<1 \\ \ln 2 & \text { if } a^{2}=1\end{cases}
$$


As one would expect, the coefficient $\alpha$ vanishes if $A A^{*}<I$ or $A A^{*}>I$. This follows from identity (5.14). If $A A^{*}>I$ then the constant $\gamma_{n-2}$ gives no contribution, again by (5.14) and

$$
\lim _{\varepsilon \rightarrow 0} R_{\varepsilon}\left(A, A^{*}\right)=(n-1) \sum_{j=1}^{n}\left(1-a_{j}^{2}\right)^{n-2} \ln \frac{a_{j}^{2}}{a_{j}^{2}-1} \prod_{k \neq j} \frac{1}{a_{k}^{2}-a_{j}^{2}}=\int_{0}^{1} \frac{d \sigma_{n}(t)}{\operatorname{det}\left(A A^{*}-t I\right)} .
$$

Similarly, if $A A^{*}<I$ then

$$
\lim _{\varepsilon \rightarrow 0} R_{\varepsilon}\left(A, A^{*}\right)=(n-1) \sum_{j=1}^{n}\left(1-a_{j}^{2}\right)^{n-2} \ln \left(1-a_{j}^{2}\right) \prod_{k \neq j} \frac{1}{a_{k}^{2}-a_{j}^{2}}=\int_{0}^{1} \frac{d \sigma_{n}(t)}{\operatorname{det}\left(I-t A A^{*}\right)} .
$$

Thus, (5.8) indeed reproduces formulas of Theorem 11 part (ii).

\section{Rank-one perturbations of CUE and GUE}

In this section we express the mean eigenvalue density for the random matrix ensembles (1.4) and (1.6) in terms of the spectral determinants. Our calculation is inspired by similar calculations in [17, 18 and makes use of a process known as eigenvalue deflation which was introduced in the context of random matrices in [44].

We need to recall a few facts about elementary unitary Hermitian matrices [47. Let $\boldsymbol{v}$ be a column-vector in $\mathbb{C}^{n}$. The matrix

$$
R_{\boldsymbol{v}}=I_{n}-2 \boldsymbol{v} \boldsymbol{v}^{*} /|\boldsymbol{v}|^{2}, \quad|\boldsymbol{v}|^{2}=\boldsymbol{v}^{*} \boldsymbol{v}
$$

where $I_{n}$ is identity matrix, defines a linear transformation which is reflection across the hyperplane through the origin with normal $\boldsymbol{v} /|\boldsymbol{v}|$. It is straightforward to verify that $R_{\boldsymbol{v}}$ is unitary and Hermitian,

$$
R_{\boldsymbol{v}}=R_{\boldsymbol{v}}^{*} \text { and } R_{\boldsymbol{v}} R_{\boldsymbol{v}}^{*}=R_{\boldsymbol{v}}^{2}=I_{n}
$$

In the context of numerical linear algebra the matrices $R_{\boldsymbol{v}}$ are known as Householder reflections. Any matrix can be brought to triangular form by a succession of Householder reflections. We only need the first step of this process which we now describe.

Let $W_{n}$ be an $n \times n$ matrix and $z$ and $\boldsymbol{x}=\left(x_{1}, \ldots, x_{n}\right)^{T}$ be an eigenvalue and eigenvector of $W_{n}$, so that

$$
W_{n} \boldsymbol{x}=z \boldsymbol{x} .
$$

Without loss of generality we may assume that $x_{1} \geq 0$ and $|\boldsymbol{x}|^{2}=\boldsymbol{x}^{*} \boldsymbol{x}=1$. Let $\boldsymbol{e}_{\mathbf{1}}=$ $(1,0, \ldots, 0)^{T}$ and

$$
\boldsymbol{v}=\frac{\boldsymbol{x}+\boldsymbol{e}_{\mathbf{1}}}{\left|\boldsymbol{x}+\boldsymbol{e}_{\mathbf{1}}\right|}=\frac{\boldsymbol{x}+\boldsymbol{e}_{\mathbf{1}}}{\sqrt{2\left(1+x_{1}\right)}}
$$


Since the vector $\boldsymbol{v}$ bisects the angle of $\boldsymbol{x}$ and $\boldsymbol{e}_{1}$, we have $R_{\boldsymbol{v}} \boldsymbol{x}=-\boldsymbol{e}_{\mathbf{1}}$ and $R_{\boldsymbol{v}} \boldsymbol{e}_{\mathbf{1}}=-\boldsymbol{x}$. Therefore $R_{\boldsymbol{v}} W_{n} R_{\boldsymbol{v}} \boldsymbol{e}_{\mathbf{1}}=z \boldsymbol{e}_{\mathbf{1}}$ and (recall that $R_{\boldsymbol{v}}^{2}=I_{n}$ )

$$
W_{n}=R_{\boldsymbol{v}}\left(\begin{array}{cc}
z & \boldsymbol{w}^{*} \\
\mathbf{0} & W_{n-1}
\end{array}\right) R_{\boldsymbol{v}}
$$

for some $W_{n-1}$ and $\boldsymbol{w}$. Note that $W_{n-1}$ is $(n-1) \times(n-1)$ and $\boldsymbol{w}^{*}$ is $1 \times(n-1)$. Obviously, applying this procedure again (to the matrix $W_{n-1}$ ) and again, one can reduce $W_{n}$ to triangular form by means of unitary transformations. Such factorization is known as Schur decomposition.

It is convenient to write $\boldsymbol{v}=\left(v_{1}, \boldsymbol{q}\right)^{T}$, where $\boldsymbol{q}=\left(v_{2}, \ldots, v_{n}\right)^{T}$. Since $\boldsymbol{v}$ is a unit vector, $v_{1}^{2}+|\boldsymbol{q}|^{2}=1$. Note that the first equation in (6.1) reads $v_{1}=\sqrt{\left(1+x_{1}\right) / 2}$. Since $0 \leq x_{1} \leq 1$, we must have $1 / 2 \leq v_{1} \leq 1$. Therefore,

$$
v_{1}=\sqrt{1-|\boldsymbol{q}|^{2}} \text { and } \frac{1}{2} \leq|\boldsymbol{q}|^{2} \leq 1
$$

In terms of $\boldsymbol{q}$ the matrix $R_{\boldsymbol{v}}$ is given by

$$
R_{\boldsymbol{v}}=\left(\begin{array}{cc}
1-2 v_{1}^{2} & -2 v_{1} \boldsymbol{q}^{*} \\
-2 v_{1} \boldsymbol{q} & I_{n-1}-2 \boldsymbol{q} \boldsymbol{q}^{*}
\end{array}\right)=\left(\begin{array}{cc}
2|\boldsymbol{q}|^{2}-1 & -2 \sqrt{1-|\boldsymbol{q}|^{2}} \boldsymbol{q}^{*} \\
-2 \sqrt{1-|\boldsymbol{q}|^{2}} \boldsymbol{q} & I_{n-1}-2 \boldsymbol{q} \boldsymbol{q}^{*}
\end{array}\right)
$$

The incomplete Schur decomposition (6.2) gives rise to a new coordinate system in the space of complex matrices, the new (complex) coordinates being $z, \boldsymbol{w}, \boldsymbol{q}$ and the matrix entries of $W_{n-1}$. There are no restrictions on the range of variation of $z, \boldsymbol{w}$ and $W_{n-1}$, and, in view of (6.3), the vector $\boldsymbol{q}$ is restricted to the spherical segment $\frac{1}{2} \leq|\boldsymbol{q}|^{2} \leq 1$. The Jacobian of the transformation from $\left(W_{n, j k}\right)$ to this new system of coordinates ${ }^{3}$,

$$
\begin{aligned}
& \prod_{j, k=1}^{n} \frac{d\left(W_{n}\right)_{j k} d \overline{\left(W_{n}\right)_{j k}}}{2}= \\
& \quad J\left(z, \boldsymbol{w}, \boldsymbol{q}, W_{n-1}\right) \frac{d z d \bar{z}}{2} \prod_{j=1}^{n-1} \frac{d w_{j} d \overline{w_{j}}}{2} \prod_{j=1}^{n-1} \frac{d q_{j} d \overline{q_{j}}}{2} \prod_{j, k=1}^{n-1} \frac{d\left(W_{n-1}\right)_{j k} d \overline{\left(W_{n-1}\right)_{j k}}}{2},
\end{aligned}
$$

is given by (cf. Lemma 3.2 in [18])

$$
J\left(z, \boldsymbol{w}, \boldsymbol{q}, W_{n-1}\right)=2^{2 n-2}\left|\operatorname{det}\left(z I_{n-1}-W_{n-1}\right)\right|^{2}\left(1-|\boldsymbol{q}|^{2}\right)^{n-2}\left(2|\boldsymbol{q}|^{2}-1\right) .
$$

We derive (6.5) in Appendix B.

Suppose that we have a probability distribution

$$
d P\left(W_{n}\right)=p\left(W_{n}\right) \prod_{i, j=1}^{n} \frac{d\left(W_{n}\right)_{i j} d \overline{\left(W_{n}\right)_{i j}}}{2}
$$

\footnotetext{
${ }^{3}$ For Jacobian computations it is convenient to consider $z$ and $\bar{z}$ as functionally independent variables, so that $d^{2} z \equiv d \operatorname{Re} z d \operatorname{Im} z=d z d \bar{z} / 2$.
} 
on the space of complex $n \times n$ matrices. Then, following the argument of [18], see their Lemma 3.1, the mean eigenvalue density $\rho_{n}(x, y), z=x+i y$, of $W_{n}$ is given by

$$
\rho_{n}(x, y)=\int_{\mathbb{C}^{2(n-1)^{2}}} d^{2} W_{n-1} \int_{\mathbb{C}^{n-1}} d^{2} \boldsymbol{w} \int_{\frac{1}{2} \leq|\boldsymbol{q}|^{2} \leq 1} d^{2} \boldsymbol{q} J\left(z, \boldsymbol{w}, \boldsymbol{q}, W_{n-1}\right) p\left(R_{\boldsymbol{v}}\left(\begin{array}{cc}
z & \boldsymbol{w}^{*} \\
\mathbf{0} & W_{n-1}
\end{array}\right) R_{\boldsymbol{v}}\right),
$$

where

$$
d^{2} W_{n-1}=\prod_{j, k=1}^{n-1} \frac{d\left(W_{n-1}\right)_{j k} d \overline{\left(W_{n-1}\right)_{j k}}}{2}, \quad d^{2} \boldsymbol{q}=\prod_{j=1}^{n-1} \frac{d q_{j} d \overline{q_{j}}}{2}, \quad d^{2} \boldsymbol{w}=\prod_{j=1}^{n-1} \frac{d w_{j} d \overline{w_{j}}}{2} .
$$

Since we integrate in the $\boldsymbol{q}$-space over the spherical segment $1 / 2 \leq|\boldsymbol{q}|^{2} \leq 1$, it is convenient to introduce spherical coordinates,

$$
\boldsymbol{q}=\sqrt{t} \boldsymbol{\sigma}, \quad t=|\boldsymbol{q}|^{2}, \quad \boldsymbol{\sigma}=\boldsymbol{q} /|\boldsymbol{q}|
$$

The element of volume in the $\boldsymbol{q}$-space is then

$$
d^{2} \boldsymbol{q}=\frac{1}{2} t^{n-2} d t d S(\boldsymbol{\sigma})
$$

where $d S(\boldsymbol{\sigma})$ is the element of area of the sphere $|\boldsymbol{\sigma}|^{2}=1$. The range of variation of $t$ is $1 / 2 \leq t \leq 1$. Next, on making the substitution

$$
r=(2 t-1)^{2}, \quad(2 t-1) d t=\frac{1}{4} d r, \quad(1-t) t=\frac{1}{4}(1-r), \quad t=\frac{1+\sqrt{r}}{2},
$$

the expression for the Jacobian becomes simpler,

$$
\begin{aligned}
J\left(z, \boldsymbol{w}, \boldsymbol{q}, W_{n-1}\right) d^{2} \boldsymbol{q} & =J\left(z, \boldsymbol{w}, \sqrt{t} \boldsymbol{\sigma}, W_{n-1}\right) d^{2} \boldsymbol{q} \\
& =2^{2 n-3}\left|\operatorname{det}\left(z I_{n-1}-W_{n-1}\right)\right|^{2}[(1-t) t]^{n-2}(2 t-1) d t d S(\boldsymbol{\sigma}) \\
& =\frac{1}{2}\left|\operatorname{det}\left(z I_{n-1}-W_{n-1}\right)\right|^{2}(1-r)^{n-2} d r d S(\boldsymbol{\sigma}) .
\end{aligned}
$$

Substituting this into (6.7), we arrive at the desired formula for the mean density of eigenvalues in the ensemble with matrix distribution (6.6):

$$
\rho_{n}(x, y)=\frac{1}{2} \int_{\mathbb{C}^{2(n-1)^{2}}} d^{2} W_{n-1} \int_{\mathbb{C}^{n-1}} d^{2} \boldsymbol{w} \int_{|\boldsymbol{\sigma}|^{2}=1} d S(\boldsymbol{\sigma}) \int_{0}^{1}(1-r)^{n-2} d r\left|\operatorname{det}\left(z I_{n-1}-W_{n-1}\right)\right|^{2} p\left(R\left(\begin{array}{cc}
z & \boldsymbol{w}^{*} \\
\mathbf{0} & W_{n-1}
\end{array}\right) R\right) .
$$

Here

$$
R=\left(\begin{array}{cc}
\sqrt{r} & \sqrt{1-r} \boldsymbol{\sigma}^{*} \\
-\sqrt{1-r} \boldsymbol{\sigma} & I_{n-1}-(1+\sqrt{r}) \boldsymbol{\sigma} \boldsymbol{\sigma}^{*}
\end{array}\right)
$$


We shall now apply this result to express the mean density of eigenvalues in terms of the absolute square modulus of characteristic polynomials for two ensembles of random matrices.

Rank-one deviations from unitarity. Let $U_{n}$ be an $n \times n$ unitary matrix and

$$
G_{n}=\left(\begin{array}{cc}
\sqrt{1-\gamma} & 0 \\
0 & I_{n-1}
\end{array}\right), \quad 0 \leq \gamma \leq 1
$$

The Haar measure on $U(n)$ induces a measure on the manifold $W_{n}^{*} W_{n}=G_{n}^{2}$ in the space of complex $n \times n$ matrices via the correspondence $W_{n}=U_{n} G_{n}$. The corresponding matrix distribution is uniform and can be conveniently described via matrix delta-function

$$
d P\left(W_{n}\right)=c_{n} \delta\left(W_{n}^{*} W_{n}-G_{n}^{2}\right) \prod_{i, j=1}^{n} \frac{d\left(W_{n}\right)_{i j} d \overline{\left(W_{n}\right)_{i j}}}{2}
$$

For Hermitian $H$, we define $\delta(H)$ as

$$
\delta(H)=\prod_{j} \delta\left(H_{j j}\right) \prod_{j<l} \delta\left(H_{j k}\right) \delta\left(\overline{H_{j k}}\right) .
$$

The normalization constant $c_{n}$ can be easily computed by changing to the matrix 'polar' coordinates,

$$
c_{n}=\frac{2^{n}}{\operatorname{Vol}(U(n))}=\frac{1 ! 2 ! \cdots(n-1) !}{\pi^{n(n+1) / 2}} .
$$

Note that the eigenvalues of $G_{n} U_{n}$ and $U_{n} G_{n}$ coincide, and, therefore, for the purpose of calculating the eigenvalue statistics the ensembles $G_{n} U_{n}$ and $U_{n} G_{n}$ are equivalent. In view of (6.2) it is more convenient to work with matrices $U_{n} G_{n}$.

Changing the coordinate system to $z, \boldsymbol{w}, r, \boldsymbol{\sigma}, W_{n-1}$,

$$
\begin{aligned}
d P\left(W_{n}\right)= & \frac{c_{n}}{2} \delta\left(\left(\begin{array}{cc}
\bar{z} & \mathbf{0} \\
\boldsymbol{w} & W_{n-1}^{*}
\end{array}\right)\left(\begin{array}{cc}
z & \boldsymbol{w}^{*} \\
\mathbf{0} & W_{n-1}
\end{array}\right)-R G_{n}^{2} R\right) \times \\
& \left|\operatorname{det}\left(z I_{n-1}-W_{n-1}\right)\right|^{2} d^{2} z(1-r)^{n-2} d r d S(\boldsymbol{\sigma}) d^{2} \boldsymbol{w} d^{2} W_{n-1},
\end{aligned}
$$

where we have used the unitary invariance of the matrix delta-function.

The matrix inside the delta-function in (6.14) is

$$
\left(\begin{array}{cc}
|z|^{2}-1+\gamma r & \bar{z} \boldsymbol{w}^{*}-\gamma \sqrt{(1-r) r} \boldsymbol{\sigma}^{*} \\
z \boldsymbol{w}-\gamma \sqrt{(1-r) r} \boldsymbol{\sigma} & W_{n-1}^{*} W_{n-1}-I_{n-1}+\boldsymbol{w} \boldsymbol{w}^{*}+\gamma(1-r) \boldsymbol{\sigma} \boldsymbol{\sigma}^{*}
\end{array}\right)
$$

and the delta-function factorizes into the product of the three delta-functions, correspondingly. On substituting this into (6.8) we obtain

$$
\rho_{n}(x, y)=\frac{c_{n}}{2} \int_{\mathbb{C}^{2(n-1)^{2}}}\left|\operatorname{det}\left(z I_{n-1}-W_{n-1}\right)\right|^{2} f\left(W_{n-1}\right) d^{2} W_{n-1}
$$


where

$$
\begin{aligned}
f\left(W_{n-1}\right)= & \int_{|\boldsymbol{\sigma}|^{2}=1} d S(\boldsymbol{\sigma}) \int_{0}^{1}(1-r)^{n-2} d r \delta\left(|z|^{2}-1+\gamma r\right) \times \\
& \int_{\mathbb{C}^{n-1}} d^{2} \boldsymbol{w} \delta\left(W_{n-1}^{*} W_{n-1}-I_{n-1}+\boldsymbol{w} \boldsymbol{w}^{*}+\gamma(1-r) \boldsymbol{\sigma} \boldsymbol{\sigma}^{*}\right) \delta(z \boldsymbol{w}-\gamma \sqrt{(1-r) r} \boldsymbol{\sigma}) .
\end{aligned}
$$

Note that

$$
\delta(z \boldsymbol{w}-\gamma \sqrt{(1-r) r} \boldsymbol{\sigma})=\frac{1}{|z|^{2(n-1)}} \delta\left(\boldsymbol{w}-\frac{\gamma \sqrt{(1-r) r} \boldsymbol{\sigma}}{z}\right) .
$$

Therefore the integral over $\boldsymbol{w}$ yields

$$
\frac{1}{|z|^{2(n-1)}} \delta\left(W_{n-1}^{*} W_{n-1}-I_{n-1}+\frac{\gamma(1-r)\left(\gamma r+|z|^{2}\right)}{|z|^{2}} \boldsymbol{\sigma} \boldsymbol{\sigma}^{*}\right)
$$

and

$$
\begin{aligned}
& f\left(W_{n-1}\right)=\frac{1}{|z|^{2(n-1)}} \int_{|\boldsymbol{\sigma}|^{2}=1} d S(\boldsymbol{\sigma}) \int_{0}^{1}(1-r)^{n-2} d r \delta\left(|z|^{2}-1+\gamma r\right) \times \\
& \delta\left(W_{n-1}^{*} W_{n-1}-I_{n-1}+\frac{\gamma(1-r)\left(\gamma r+|z|^{2}\right)}{|z|^{2}} \boldsymbol{\sigma}^{*}\right) .
\end{aligned}
$$

It is apparent that if $|z|^{2}<1-\gamma$ or $|z|^{2}>1$ then the integral over $r$ vanishes and $f\left(W_{n-1}\right)=0$. Therefore $\rho_{n}(x, y)=0$ for such values of $z$. If $1-\gamma<|z|^{2}<1$ then the integral over $r$ produces a non-trivial contribution and

$$
f\left(W_{n-1}\right)=\frac{1}{\gamma|z|^{2(n-1)}} \int_{|\boldsymbol{\sigma}|^{2}=1} d S(\boldsymbol{\sigma})\left(1-\frac{1-|z|^{2}}{\gamma}\right)^{n-2} \delta\left(W_{n-1}^{*} W_{n-1}-I_{n-1}+\frac{\gamma-1+|z|^{2}}{|z|^{2}} \boldsymbol{\sigma} \boldsymbol{\sigma}^{*}\right) .
$$

Introducing

$$
\tilde{\gamma}=\frac{\gamma-1+|z|^{2}}{|z|^{2}}
$$

we can rewrite the above expression in a shorter form,

$$
f\left(W_{n-1}\right)=\frac{1}{\gamma|z|^{2}}\left(\frac{\tilde{\gamma}}{\gamma}\right)^{n-2} \int_{|\boldsymbol{\sigma}|^{2}=1} d S(\boldsymbol{\sigma}) \delta\left(W_{n-1}^{*} W_{n-1}-I_{n-1}+\tilde{\gamma} \boldsymbol{\sigma} \boldsymbol{\sigma}^{*}\right) .
$$

On substituting this into (6.15), we arrive at

$$
\begin{gathered}
\rho_{n}(x, y)=\frac{c_{n}}{2 \gamma|z|^{2}}\left(\frac{\tilde{\gamma}}{\gamma}\right)^{n-2} \int_{\mathbb{C}^{2(n-1)^{2}}} d^{2} W_{n-1}\left|\operatorname{det}\left(z I_{n-1}-W_{n-1}\right)\right|^{2} \times \\
\int_{|\boldsymbol{\sigma}|^{2}=1} d S(\boldsymbol{\sigma}) \delta\left(W_{n-1}^{*} W_{n-1}-I_{n-1}+\tilde{\gamma} \boldsymbol{\sigma} \boldsymbol{\sigma}^{*}\right) .
\end{gathered}
$$


Since the matrix $\boldsymbol{\sigma} \boldsymbol{\sigma}^{*}$ is unitary equivalent to the matrix

$$
\left(\begin{array}{cc}
1 & \mathbf{0} \\
\mathbf{0} & 0_{n-1}
\end{array}\right)
$$

the integration over $\boldsymbol{\sigma}$ can be easily performed yielding

$$
\rho_{n}(x, y)=\frac{c_{n} \operatorname{Vol}\left(S^{2 n-3}\right)}{2 \gamma|z|^{2}}\left(\frac{\tilde{\gamma}}{\gamma}\right)_{\mathbb{C}^{2(n-1)^{2}}}^{n-2}\left|\operatorname{det}\left(z I_{n-1}-W_{n-1}\right)\right|^{2} \delta\left(W_{n-1}^{*} W_{n-1}-\tilde{G}_{n-1}^{2}\right) d^{2} W_{n-1},
$$

where $\tilde{G}_{n-1}$ is the $(n-1) \times(n-1)$ matrix $($ cf. (6.10) $)$

$$
\tilde{G}_{n-1}=\left(\begin{array}{cc}
\sqrt{1-\tilde{\gamma}} & \mathbf{0} \\
\mathbf{0} & I_{n-2}
\end{array}\right)
$$

and $\operatorname{Vol}\left(S^{2 n-3}\right)$ is the area of the unit sphere in $\mathbb{R}^{2(n-1)}$,

$$
\operatorname{Vol}\left(S^{2 n-3}\right)=\frac{2 \pi^{n-1}}{(n-2) !} .
$$

It follows from (6.13) that $c_{n} / c_{n-1}=(n-1) ! / \pi^{n}$. Hence

$$
\frac{c_{n} \operatorname{Vol}\left(S^{2 n-3}\right)}{2}=\frac{n-1}{\pi} c_{n-1},
$$

and finally

$$
\begin{aligned}
\rho_{n}(x, y) & =\frac{n-1}{\pi \gamma|z|^{2}}\left(\frac{\tilde{\gamma}}{\gamma}\right)^{n-2} c_{n-1} \int_{\mathbb{C}^{2(n-1)^{2}}}\left|\operatorname{det}\left(z I_{n-1}-W_{n-1}\right)\right|^{2} \delta\left(W_{n-1}^{*} W_{n-1}-\tilde{G}_{n-1}^{2}\right) d^{2} W_{n-1} \\
& =\frac{n-1}{\pi \gamma|z|^{2}}\left(\frac{\tilde{\gamma}}{\gamma}\right)^{n-2} \int_{U(n-1)}\left|\operatorname{det}\left(z I_{n-1}-U_{n-1} \tilde{G}_{n-1}\right)\right|^{2} d U_{n-1}
\end{aligned}
$$

as claimed in (1.5).

Rank-one deviations from Hermiticity. Let $H_{n}$ be a $\mathrm{GUE}_{n}$ matrix, i.e. random Hermitian matrix of size $n \times n$ with probability distribution

$$
c_{\beta, n} e^{-\frac{\beta}{2} \operatorname{tr} H_{n}^{2}} \prod_{j=1}^{n} d\left(H_{n}\right)_{j j} \prod_{1 \leq j<k \leq n} \frac{d\left(H_{n}\right)_{j j} d \overline{\left(H_{n}\right)_{j j}}}{2}, \quad \beta>0,
$$

and $\Gamma_{n}$ be the $n \times n$ matrix

$$
\Gamma_{n}=\gamma\left(\begin{array}{cc}
1 & 0 \\
0 & 0_{n-1}
\end{array}\right), \quad \gamma>0
$$


Consider the random matrices

$$
W_{n}=H_{n}+i \Gamma_{n}
$$

Obviously,

$$
\operatorname{Re} W_{n}:=\frac{W_{n}+W_{n}^{*}}{2}=H_{n}, \quad \text { and } \quad \operatorname{Im} W_{n}:=\frac{W_{n}-W_{n}^{*}}{2 i}=\Gamma_{n} .
$$

The matrices $W_{n}$ are complex and their probability distribution is given by

$$
d P\left(W_{n}\right)=c_{\beta, n} e^{-\frac{\beta}{2} \operatorname{tr}\left(\operatorname{Re} W_{n}\right)^{2}} \delta\left(\operatorname{Im} W_{n}-\Gamma_{n}\right) \prod_{i, j=1}^{n} \frac{d\left(W_{n}\right)_{i j} d \overline{\left(W_{n}\right)_{i j}}}{2},
$$

where $c_{\beta, n}$ is the normalization constant,

$$
c_{\beta, n}=\left(\frac{1}{2}\right)^{n / 2}\left(\frac{\beta}{\pi}\right)^{n^{2} / 2}
$$

and $\delta$ is the matrix delta-function (6.12).

Changing the coordinate system to $z, \boldsymbol{w}, r, \boldsymbol{\sigma}$ and $W_{n-1}$,

$$
\begin{aligned}
d P\left(W_{n}\right)= & \frac{1}{2} c_{\beta, n} e^{-\frac{\beta}{2} \operatorname{tr}\left(\operatorname{Re} W_{n-1}\right)^{2}-\frac{\beta}{4}|\boldsymbol{w}|^{2}-\frac{\beta}{2}(\operatorname{Re} z)^{2} \delta}\left(\left(\begin{array}{cc}
\operatorname{Im} z & \frac{\boldsymbol{w}^{*}}{2 i} \\
-\frac{\boldsymbol{w}}{2 i} & \operatorname{Im} W_{n-1}
\end{array}\right)-R \Gamma_{n} R\right) \times(6.1) \\
& \left|\operatorname{det}\left(z I_{n-1}-W_{n-1}\right)\right|^{2} d^{2} z(1-r)^{n-2} d r d S(\boldsymbol{\sigma}) d^{2} \boldsymbol{w} d^{2} W_{n-1}
\end{aligned}
$$

where we have used the unitary invariance of the matrix delta-function.

The matrix inside the delta-function in (6.14) is

$$
\left(\begin{array}{cc}
\operatorname{Im} z-\gamma r & \frac{\boldsymbol{w}^{*}}{2 i}+\frac{\gamma}{2} \sqrt{(1-r) r} \boldsymbol{\sigma}^{*} \\
-\frac{\boldsymbol{w}^{*}}{2 i}+\frac{\gamma}{2} \sqrt{(1-r) r} \boldsymbol{\sigma}^{*} & \operatorname{Im} W_{n-1}-\gamma(1-r) \boldsymbol{\sigma} \boldsymbol{\sigma}^{*}
\end{array}\right)
$$

and the delta-function factorizes into the product of the three delta-functions correspondingly. On substituting this into (6.8) we obtain

$$
\rho_{n}(x, y)=\frac{1}{2} c_{\beta, n} e^{-\frac{\beta x^{2}}{2}} \int_{\mathbb{C}^{2}(n-1)^{2}}\left|\operatorname{det}\left(z I_{n-1}-W_{n-1}\right)\right|^{2} f\left(W_{n-1}\right) e^{-\frac{\beta}{2} \operatorname{tr}\left(\operatorname{Re} W_{n-1}\right)^{2}} d^{2} W_{n-1}
$$

where

$$
\begin{array}{r}
f\left(W_{n-1}\right)=\int_{|\boldsymbol{\sigma}|^{2}=1} d S(\boldsymbol{\sigma}) \int_{0}^{1}(1-r)^{n-2} d r \delta(y-\gamma r) \delta\left(\operatorname{Im} W_{n-1}-\gamma(1-r) \boldsymbol{\sigma} \boldsymbol{\sigma}^{*}\right) \times \\
\int_{\mathbb{C}^{n-1}} d^{2} \boldsymbol{w} e^{-\frac{\beta|\boldsymbol{w}|^{2}}{4}} \delta\left(\frac{\boldsymbol{w}^{*}}{2 i}+\frac{\gamma}{2} \sqrt{(1-r) r} \boldsymbol{\sigma}^{*}\right) .
\end{array}
$$


The integral over $\boldsymbol{w}$ yields $\frac{1}{4} e^{-\beta \gamma^{2}(1-r) r}$, and we arrive at

$$
f\left(W_{n-1}\right)=\frac{1}{4} \int_{|\boldsymbol{\sigma}|^{2}=1} d S(\boldsymbol{\sigma}) \int_{0}^{1} d r(1-r)^{n-2} e^{-\beta \gamma^{2}(1-r) r} \delta(y-\gamma r) \delta\left(\operatorname{Im} W_{n-1}-\gamma(1-r) \boldsymbol{\sigma} \boldsymbol{\sigma}^{*}\right) .
$$

It is apparent that if $y<0$ or $y>\gamma$ then the integral over $r$ vanishes. Therefore $\rho_{n}(x, y)=0$ if $y<0$ or $y>\gamma$. If $0<y<\gamma$, then the integration over $r$ produces the factor $\frac{1}{\gamma}$ and the constraint $r=\frac{y}{\gamma}$, so that

$$
f\left(W_{n-1}\right)=\frac{(\gamma-y)^{n-2} e^{-\beta(\gamma-y) y}}{4 \gamma^{n-1}} \int_{|\boldsymbol{\sigma}|^{2}=1} d S(\boldsymbol{\sigma}) \delta\left(\operatorname{Im} W_{n-1}-(\gamma-y) \boldsymbol{\sigma} \boldsymbol{\sigma}^{*}\right) .
$$

On substituting the obtained expression for $f\left(W_{n-1}\right)$ into (6.20), we arrive at

$$
\begin{aligned}
\rho_{n}(x, y)= & \frac{c_{\beta, n}(\gamma-y)^{n-2} e^{-\frac{\beta x^{2}}{2}-\beta(\gamma-y) y}}{8 \gamma^{n-1}} \int_{\mathbb{C}^{2(n-1)^{2}}} d^{2} W_{n-1}\left|\operatorname{det}\left(z I_{n-1}-W_{n-1}\right)\right|^{2} e^{-\frac{\beta}{2} \operatorname{tr}\left(\operatorname{Re}\left(W_{n-1}\right)^{2}\right.} \times \\
& \int_{|\boldsymbol{\sigma}|^{2}=1} d S(\boldsymbol{\sigma}) \delta\left(\operatorname{Im} W_{n-1}-(\gamma-y) \boldsymbol{\sigma} \boldsymbol{\sigma}^{*}\right) .
\end{aligned}
$$

The integral over $\boldsymbol{\sigma}$ yields

$$
\operatorname{Vol}\left(S^{2 n-3}\right) \delta\left(\operatorname{Im} W_{n-1}-\tilde{\Gamma}_{n-1}\right)
$$

where $\tilde{\Gamma}_{n-1}$ is the $(n-1) \times(n-1)$ matrix

$$
\tilde{\Gamma}_{n-1}=(\gamma-y)\left(\begin{array}{cc}
1 & \mathbf{0} \\
\mathbf{0} & 0_{n-2}
\end{array}\right)
$$

and $\operatorname{Vol}\left(S^{2 n-3}\right)$ is the area of the unit sphere in $\mathbb{R}^{2(n-1)}$ (6.16). We have that

$$
\frac{c_{\beta, n}}{c_{\beta, n-1}}=\left(\frac{\beta}{\pi}\right)^{n}\left(\frac{\pi}{2 \beta}\right)^{1 / 2}
$$

and it now follows that

$$
\begin{aligned}
\rho_{n}(x, y)= & \frac{1}{4 \sqrt{2 \pi \beta}} \frac{\beta^{n}}{(n-2) !} \frac{(\gamma-y)^{n-2} e^{-\frac{\beta x^{2}}{2}-\beta(\gamma-y) y}}{\gamma^{n-1}} \times \\
& c_{\beta, n-1} \int_{\mathbb{C}^{2(n-1)^{2}}} d^{2} W_{n-1}\left|\operatorname{det}\left(z I_{n-1}-W_{n-1}\right)\right|^{2} e^{-\frac{\beta}{2} \operatorname{tr}\left(\operatorname{Re}\left(W_{n-1}\right)^{2}\right.} \delta\left(\operatorname{Im} W_{n-1}-\tilde{\Gamma}_{n-1}\right),
\end{aligned}
$$

as claimed in (1.7). 


\section{A Appendix}

In this appendix we evaluate the integral

$$
\mathcal{I}_{k}\left(\varepsilon^{2}, a^{2}\right)=\int_{0}^{1} \frac{(1-t)^{k} d t}{\sqrt{\left(t-a^{2}+\varepsilon^{2}\right)^{2}+4 \varepsilon^{2} a^{2}}}
$$

in the limit $\varepsilon \rightarrow 0$.

We shall use the following fact from Calculus. If $P(t)$ is a polynomial of degree $k$ then (integrate by parts)

$$
\int \frac{P(t) d t}{\sqrt{t^{2}+p t+q}}=Q(t) \sqrt{t^{2}+p t+q}+\lambda \int \frac{d t}{\sqrt{t^{2}+p t+q}}
$$

where $Q$ is a polynomial of degree $k-1$ and $\lambda$ is a constant. For $Q$ and $\lambda$ one has the equation (differentiate (A.1))

$$
P(t)=Q^{\prime}(t)\left(t^{2}+p t+q\right)+\frac{1}{2} Q(t)\left(t^{2}+p t+q\right)^{\prime}+\lambda .
$$

It follows from this that

$$
\begin{aligned}
\mathcal{I}_{k}\left(\varepsilon^{2}, a^{2}\right) & =\left[Q_{\varepsilon, a}(t) \sqrt{\left(t-a^{2}+\varepsilon^{2}\right)^{2}+4 \varepsilon^{2} a^{2}}\right]_{t=0}^{t=1}+\lambda_{\varepsilon, a} \mathcal{I}_{0}(\varepsilon, a) \\
& =Q_{\varepsilon, a}(1) \sqrt{\left(1-a^{2}+\varepsilon^{2}\right)^{2}+4 \varepsilon^{2} a^{2}}-Q_{\varepsilon, a}(0)\left(\varepsilon^{2}+a^{2}\right)+\lambda_{\varepsilon, a} \mathcal{I}_{0}(\varepsilon, a),
\end{aligned}
$$

and the equation for $Q(t)$ and $\lambda$ is

$$
(1-t)^{k}=Q_{\varepsilon, a}^{\prime}(t)\left[\left(t-a^{2}+\varepsilon^{2}\right)^{2}+4 \varepsilon^{2} a^{2}\right]+Q_{\varepsilon, a}(t)\left(t-a^{2}+\varepsilon^{2}\right)+\lambda_{\varepsilon, a} .
$$

It is apparent from (A.2) that $Q(t)$ and $\lambda$ must be polynomials in $a^{2}$ and $\varepsilon^{2}$ and, therefore, in the limit $\varepsilon \rightarrow 0$,

$$
Q_{\varepsilon, a}(t)=Q_{a}(t)+O\left(\varepsilon^{2}\right) \text { and } \quad \lambda_{\varepsilon, a}=\lambda_{a}+O\left(\varepsilon^{2}\right),
$$

and

$$
(1-t)^{k}=Q_{a}^{\prime}(t)\left(t-a^{2}\right)^{2}+Q_{a}(t)\left(t-a^{2}\right)+\lambda_{a} .
$$

This equation for $Q_{a}$ and $\lambda_{a}$ can be explicitly solved, the solution being

$$
\lambda_{a}=\left(1-a^{2}\right)^{k} \quad \text { and } \quad Q_{a}(t)=\sum_{l=1}^{k} \frac{(-1)^{l}}{l}\left(\begin{array}{c}
k \\
l
\end{array}\right)\left(t-a^{2}\right)^{l-1}\left(1-a^{2}\right)^{k-l} \text {. }
$$

Note that at $Q_{a}(0)$ is a polynomial in $a^{2}$ of degree $k-1$, and

$$
Q_{a}(1)=\left(1-a^{2}\right)^{k-1} \sum_{l=1}^{k} \frac{(-1)^{l}}{l}\left(\begin{array}{l}
k \\
l
\end{array}\right)=-\left(1-a^{2}\right)^{k-1} \gamma_{k},
$$


where $\gamma_{k}$ is the partial sum of the harmonic series,

$$
\gamma_{k}=1+\frac{1}{2}+\ldots+\frac{1}{k}
$$

We now turn to $\mathcal{I}_{0}\left(\varepsilon^{2}, a^{2}\right)$. Recalling the table integral

$$
\int \frac{d t}{\sqrt{t^{2}+\alpha^{2}}}=\ln \left|t+\sqrt{t^{2}+\alpha^{2}}\right|
$$

we have

$$
\mathcal{I}_{0}\left(\varepsilon^{2}, a^{2}\right)=\ln \frac{1-a^{2}+\varepsilon^{2}+\sqrt{\left(1-a^{2}+\varepsilon^{2}\right)^{2}+4 \varepsilon^{2} a^{2}}}{2 \varepsilon^{2}}
$$

At $a^{2}=1$,

$$
\mathcal{I}_{0}\left(\varepsilon^{2}, 1\right)=\ln \frac{\varepsilon^{2}+\sqrt{\varepsilon^{4}+4 \varepsilon^{2}}}{2 \varepsilon^{2}}=\ln \frac{1}{\varepsilon}+O(\varepsilon) .
$$

For $a^{2} \neq 1$,

$$
\sqrt{\left(1-a^{2}+\varepsilon^{2}\right)^{2}+4 \varepsilon^{2} a^{2}}=\left|1-a^{2}\right|+\frac{\varepsilon^{2}\left(1+a^{2}\right)}{\left|1-a^{2}\right|}+O\left(\varepsilon^{4}\right)
$$

and therefore

$$
\mathcal{I}_{0}\left(\varepsilon^{2}, a^{2}\right)= \begin{cases}\ln \frac{1-a^{2}}{\varepsilon^{2}}+O\left(\varepsilon^{2}\right) & \text { if } a^{2}<1 \\ \ln \frac{a^{2}}{a^{2}-1}+O\left(\varepsilon^{2}\right) & \text { if } a^{2}>1\end{cases}
$$

After collecting all relevant terms we arrive at the desired formula

$$
\mathcal{I}_{k}\left(\varepsilon^{2}, a^{2}\right)=\left(1-a^{2}\right)^{k} \theta\left(1-a^{2}\right) \ln \frac{1}{\varepsilon^{2}}+\beta\left(a^{2}\right)+q_{k}\left(a^{2}\right)+O(\varepsilon),
$$

where $\theta$ is the Heaviside step-function,

$$
\theta(x)= \begin{cases}1 & \text { if } x>0 \\ \frac{1}{2} & \text { if } x=0 \\ 0 & \text { if } x<0\end{cases}
$$

$q_{k}$ is a polynomial of degree $k$ and

$$
\beta\left(a^{2}\right)=\operatorname{sgn}\left(a^{2}-1\right)\left(1-a^{2}\right)^{k}\left(\gamma_{k}-\ln \left|1-a^{2}\right|\right)+\theta\left(a^{2}-1\right) \ln a^{2} .
$$

We use the convention according to which the sign function, $\operatorname{sgn}(x)$, vanishes at $x=0$. 


\section{B Appendix}

In this appendix we derive equation (6.5). Let

$$
W_{n}=R\left(\begin{array}{cc}
z & \boldsymbol{w}^{*} \\
\mathbf{0} & W_{n-1}
\end{array}\right) R, \quad R=\left(\begin{array}{cc}
2 \boldsymbol{q}^{*} \boldsymbol{q}-1 & -2 \sqrt{1-\boldsymbol{q}^{*} \boldsymbol{q}} \boldsymbol{q}^{*} \\
-2 \sqrt{1-\boldsymbol{q}^{*} \boldsymbol{q}} \boldsymbol{q} & I_{n-1}-2 \boldsymbol{q} \boldsymbol{q}^{*}
\end{array}\right)
$$

When $z, \boldsymbol{w}, \boldsymbol{\eta}$ and $W_{n-1}$ get infinitesimal increments $d z, d \boldsymbol{w}, d \boldsymbol{q}$ and $d W_{n-1}$ the matrix $W_{n}$ gets increment

$$
d W_{n}=d R\left(\begin{array}{cc}
z & \boldsymbol{w}^{*} \\
0 & W_{n-1}
\end{array}\right) R+R\left(\begin{array}{cc}
d z & d \boldsymbol{w}^{*} \\
0 & d W_{n-1}
\end{array}\right) R+R\left(\begin{array}{cc}
z & \boldsymbol{w}^{*} \\
0 & W_{n-1}
\end{array}\right) d R .
$$

Since $R$ is unitary Hermitian, the matrix $R d R$ is skew-Hermitian, so that

$$
d T=R d R=\left(\begin{array}{cc}
d f & -d \boldsymbol{h}^{*} \\
d \boldsymbol{\sigma} & d T_{n-1}
\end{array}\right)
$$

for some $f, \boldsymbol{h}$ and $T_{n-1}$. Also $R d R=-(d R) R$, and it follows that

$$
\begin{aligned}
R\left(d W_{n}\right) R & =d T\left(\begin{array}{cc}
z & \boldsymbol{w}^{*} \\
0 & W_{n-1}
\end{array}\right)-\left(\begin{array}{cc}
z & \boldsymbol{w}^{*} \\
0 & W_{n-1}
\end{array}\right) d T+\left(\begin{array}{cc}
d z & d \boldsymbol{w}^{*} \\
0 & d W_{n-1}
\end{array}\right) \\
& =\left(\begin{array}{cc}
\boldsymbol{w}^{*} d \boldsymbol{h} & \boldsymbol{w}^{*} d f-z d \boldsymbol{h}^{*}-d \boldsymbol{h}^{*} W_{n-1}+\boldsymbol{w}^{*} d T_{n-1} \\
\left(z I-W_{n-1}\right) d \boldsymbol{h} & d \boldsymbol{h} \boldsymbol{w}^{*}+d T_{n-1} W_{n-1}-W_{n-1} d S_{n-1}
\end{array}\right)+\left(\begin{array}{cc}
d z & d \boldsymbol{w}^{*} \\
0 & d W_{n-1}
\end{array}\right)
\end{aligned}
$$

Let $d M=R\left(d W_{n}\right) R$. It is apparent that

$$
\prod_{j, k=1}^{n} d\left(W_{n}\right)_{j k} d \overline{\left(W_{n}\right)_{j k}}=\prod_{j, k=1}^{n} d M_{j k} d \bar{M}_{j k}
$$

On the other hand, it follows from (B.1) that

$$
\prod_{j, k=1}^{n} d M_{j k} d \bar{M}_{j k}=\left|\operatorname{det}\left(z I-W_{n-1}\right)\right|^{2} d z d \bar{z} \prod_{j=1}^{n-1} d w_{j} d \bar{w}_{j} \prod_{j=1}^{n-1} d h_{j} d \bar{h}_{j} \prod_{j, k=1}^{n-1} d\left(W_{n-1}\right)_{j k} d \overline{\left(W_{n-1}\right)_{j k}}
$$

To complete our derivation we now compute the Jacobian of the transformation from $\boldsymbol{h}$ to $\boldsymbol{q}$. Recall that $d \boldsymbol{h}$ is the $(2,1)$-entry of the matrix $d T=R d R$. A straightforward computation yields

$$
d \boldsymbol{h}=(2 a+b)\left(d \boldsymbol{q}^{*}\right) \boldsymbol{q} \boldsymbol{q}+a(d \boldsymbol{q})+b \boldsymbol{q} \boldsymbol{q}^{*}(d \boldsymbol{q}),
$$

where

$$
a=-2 \sqrt{1-\boldsymbol{q}^{*} \boldsymbol{q}}, \quad b=\frac{1-2 \boldsymbol{q}^{*} \boldsymbol{q}}{\sqrt{1-\boldsymbol{q}^{*} \boldsymbol{q}}} .
$$

Equation (B.4) can be written as

$$
d \boldsymbol{h}=\left(a I+b \boldsymbol{q} \boldsymbol{q}^{*}\right)(d \boldsymbol{q})+(2 a+b) \boldsymbol{q} \boldsymbol{q}^{T}(d \overline{\boldsymbol{q}}),
$$


and, therefore,

$$
\left(\begin{array}{l}
d \boldsymbol{q} \\
d \overline{\boldsymbol{q}}
\end{array}\right)=\left(\begin{array}{cc}
a I+b \boldsymbol{q} \boldsymbol{q}^{*} & (2 a+b) \boldsymbol{q} \boldsymbol{q}^{T} \\
(2 a+b) \overline{\boldsymbol{q}} \boldsymbol{q}^{*} & a I+b \overline{\boldsymbol{q}} \boldsymbol{q}^{T}
\end{array}\right)\left(\begin{array}{c}
d \boldsymbol{q} \\
d \overline{\boldsymbol{q}}
\end{array}\right)
$$

It now follows that

$$
\prod_{j=1}^{n-1} d h_{j} d \bar{h}_{j}=\operatorname{det}(a I+L) \prod_{j=1}^{n-1} d q_{j} d \bar{q}_{j}
$$

where $L$ is the $2(n-1) \times 2(n-1)$ matrix

$$
\left(\begin{array}{cc}
b \boldsymbol{q} \boldsymbol{q}^{*} & (2 a+b) \boldsymbol{q} \boldsymbol{q}^{T} \\
(2 a+b) \overline{\boldsymbol{q}} \boldsymbol{q}^{*} & b \overline{\boldsymbol{q}} \boldsymbol{q}^{T}
\end{array}\right)
$$

If we find the eigenvalues of $L$, we shall know $\operatorname{det}(a I+L)$.

To solve the eigenvalue problem for $L$, we observe that if $(\boldsymbol{f}, \boldsymbol{g})^{T}$ is an eigenvector of $L$ then

$$
\left\{\begin{array}{c}
b\left(\boldsymbol{q}^{*} \boldsymbol{f}\right) \boldsymbol{q}+(2 a+b)\left(\boldsymbol{q}^{T} \boldsymbol{g}\right) \boldsymbol{q}=\lambda \boldsymbol{f} \\
(2 a+b)\left(\boldsymbol{q}^{*} \boldsymbol{f}\right) \overline{\boldsymbol{q}}+b\left(\boldsymbol{q}^{T} \boldsymbol{g}\right) \overline{\boldsymbol{q}}=\lambda \boldsymbol{g}
\end{array}\right.
$$

for some $\lambda$. If $\lambda \neq 0$ we must have $\boldsymbol{f}=c_{1} \boldsymbol{q}$ and $\boldsymbol{g}=c_{2} \overline{\boldsymbol{q}}$ for some $c_{1}$ and $c_{2}$, and

$$
\left\{\begin{array}{l}
b\left(\boldsymbol{q}^{*} \boldsymbol{q}\right) c_{1}+(2 a+b)\left(\boldsymbol{q}^{*} \boldsymbol{q}\right) c_{2}=\lambda c_{1} \\
(2 a+b)\left(\boldsymbol{q}^{*} \boldsymbol{q}\right) c_{1}+b\left(\boldsymbol{q}^{*} \boldsymbol{q}\right) c_{2}=\lambda c_{2}
\end{array}\right.
$$

This reduced eigenvalue problem yields the two non-zero eigenvalues of $L$,

$$
\lambda_{1}=-2 a \boldsymbol{q}^{*} \boldsymbol{q} \text { and } \lambda_{2}=2(a+b) \boldsymbol{q}^{*} \boldsymbol{q} .
$$

It is now apparent that $\lambda=0$ is an eigenvalue of $L$ of multiplicity $2(n-2)$. This fact can be verified independently of the eigenvalue count by observing that for any vector $\boldsymbol{u}$ which is orthogonal to $\boldsymbol{q}$,

$$
L\left(\begin{array}{l}
\boldsymbol{u} \\
0
\end{array}\right)=0 \text { and } L\left(\begin{array}{c}
0 \\
\overline{\boldsymbol{u}}
\end{array}\right)=0 .
$$

It follows now that

$$
\operatorname{det}(a I+L)=a^{2(n-2)}\left(a+\lambda_{1}\right)\left(a+\lambda_{2}\right)=(-2)^{2 n-2}\left(1-\boldsymbol{q}^{*} \boldsymbol{q}\right)^{n-2}\left(1-2 \boldsymbol{q}^{*} \boldsymbol{q}\right) .
$$

Collecting (B.2) - (B.3) and (B.5 $-(\underline{B} .6)$, one arrives at $\underline{6.5}$.

\section{References}

[1] Akemann, G., Vernizzi G.: Characteristic Polynomials of Complex Random Matrix Models. Nucl.Phys. B 660, 532-556 (2003).

[2] Akemann, G., Pottier, A. Ratios of characteristic polynomials in complex matrix models.: J.Phys. A: Math and General 37, L453-L460 (2004). 
[3] Andreev, A.V., Simons, B.D.: Correlators of spectral determinants in quantum chaos. Phys. Rev. Lett. 75, 2304-2307 (1995).

[4] Balantekin, A.B.: Character expansions, Itzykson-Zuber integrals, and the QCD partition function. Phys. Rev. D(3) 62, 085017-085023 (2000).

[5] Baik, J., Deift, P. Strahov, E.: Products and ratios of characteristic polynomials of random Hermitian matrices. J. Math. Phys. 44, 3657-3670 (2003) .

[6] Berezin, F.A.: Some remarks on the Wigner distribution (in Russian). Teor. Mat. Fiz. 17, 305-318 (1973).

[7] Berezin, F.A.: Quantization in complex symmetric spaces. Math. USSR - Izv. 9, 341-379 (1976).

[8] Biane, Ph., Lehner, F.: Computation of some examples of Brown's spectral measure in free probability. ESI Preprint No. 823, 27 pages.

[9] Borodin, A., Olshanski, G., Strahov, E.: Giambelli compatible point processes. Epreprint ArXiv:math-ph/0505021.

[10] Borodin, A., Strahov, E.: Averages of characteristic polynomials in Random Matrix Theory. Commun. Pure and Applied Math., 59 (2), 161-253 (2006).

[11] Brezin, E., Hikami, S.: Characteristic polynomials of random matrices. Commun. Math. Phys. 214, 111-135 (2000).

[12] Bump, D., Gamburd, A.: On the average of characteristic polynomials from classical groups. Comm. Math. Phys., in press.

[13] Conrey, J.B., Farmer, D.W., Keating, J.P., Rubinstein, M.O., Snaith, N.C.: Autocorrelation of random matrix polynomials. Commun. Math. Phys. 237, 365-395 (2003).

[14] Conrey, J.B., Forrester, P.J., Snaith, N.C.: Averages of ratios of characteristic polynomials for the compact classical groups, IMRN 7, 397-431 (2005).

[15] Conrey, J.B., Farmer, D.W., Zirnbauer, M.R.: Howe pairs, supersymmetry, and ratios of random characteristic polynomials for the unitary groups $\mathrm{U}(\mathrm{N})$. E-preprint ArXiv:math-ph/0511024.

[16] Diaconis, P., Gamburd, A.: Random matrices, magic squares and matching polynomials.: Electron. J. Combin. 11, no. 2 (2004/05), research paper 2, 26 pp.

[17] Edelman, A.: The probability that a random real gaussian matrix has $k$ real eigenvalues, related distributions, and the Cirular law. J. Multiv. Anal. 60, 203-232 (1997). 
[18] Edelman, A., Kostlan, E., Shub, M.: How many eigenvalues of a random matrix are real? J. Amer. Math. Soc. 7, 247-267 (1994).

[19] Feinberg, J. and Zee, A., Non-Gaussian Non-Hermitean Random Matrix Theory: phase transitions and addition formalism. Nucl.Phys. B501, 643-669 (1997).

[20] Feinberg, J., Scalettar, R., Zee, A.: "Single Ring Theorem" and the Disk-Annulus Phase Transition. J.Math.Phys. 42, 5718-5740 (2001).

[21] Fyodorov, Y.V.: Negative moments of characteristic polynomials of random matrices: Ingham-Siegel integral as an alternative to Hubbard-Stratonovich transformation. Nuclear Phys. B 621, 643-674 (2002).

[22] Fyodorov, Y.V., Akemann, G.: On the supersymmetric partition function in QCDinspired random matrix models, JETP Lett. 77, 438-441 (2003).

[23] Fyodorov, Y.V., Khoruzhenko, B.A.: Systematic analytical approach to correlation functions of resonances in quantum chaotic scattering. Phys. Rev. Let. 83, 65-68 (1999).

[24] Fyodorov, Y.V., Sommers, H.-J.: Statistics of resonance poles, phase shifts and time delays in quantum chaotic scattering: Random matrix approach for systems with broken time-reversal invariance. J. Math. Phys. 38, 1918-1981 (1997).

[25] Fyodorov, Y.V., Sommers, H.-J.: Random matrices close to Hermitian or unitary: overview of methods and results. J. Phys. A 36, 3303-3347 (2003).

[26] Fyodorov, Y.V., Strahov E.: An exact formula for general spectral correlation function of random Hermitian matrices. J. Phys. A 36, 3203-3213 (2003).

[27] Fyodorov, Y.V., Strahov, E.: Characteristic polynomials of random Hermitian matrices and Duistermaat-Heckman localisation on non-compact Kähler Manifolds. Nucl.Phys. B630, 453-491 (2002).

[28] Haagerup, U., Larsen, F.: Brown's spectral distribution measure for $R$-diagonal elements in finite von Neumann algebras. J. Fun. Analysis 176, 331-367 (2000).

[29] Halasz, M.A., Jackson, A.D., Verbaarschot, J.J.M.: Fermion determinants in matrix models of QCD at nonzero chemical potential. Phys. Rev. D56, 5140-5152 (1997).

[30] Hua, L.K.: Harmonic Analysis of Functions of Several Complex variables in the Classical Domains. Amer. Math. Soc: Providence, Rhode Island, 1963.

[31] Ginibre, J.: Statistical Ensembles of Complex, Quaternion, and Real Matrices. J. Math. Phys. 6, 440-449 (1964).

[32] Gradshtein, I.S., Ryzhik, I.M.: Table of Integrals, Series, and Products, 5th ed., A. Jeffrey, editor: Academic Press, 1994. 
[33] Kadell, K.W.J.: The Selberg-Jack symmetric functions. Advances in Math. 130, 33-102 (1997).

[34] Kaneko, J.: Selberg integrals and hypergeometric functions associated with Jack polynomials. SIAM Jour. Math. Anal. 24, 1086-1110 (1993).

[35] Keating, J.P., Snaith, N.C.: Random matrix theory and $\zeta(1 / 2+i t))$. Comm. Math. Phys. 214, 57-89 (2000).

[36] Keating, J. P., Snaith, N. C.: Random matrix theory and $L$-functions at $s=1 / 2$. Comm. Math. Phys. 214, 91-110 (2000).

[37] Macdonald, I.G., Symmetric Functions and Hall Polynomials. 2nd ed., Clarendon Press: Oxford, 1995.

[38] Mehta, M.L.: Random matrices. 3rd ed, Elsevier/Academic Press: Amsterdam, 2004.

[39] Orlov, A. Yu.: New Solvable Matrix Integrals. In Proceedings of 6th International Workshop on Conformal Field Theory and Integrable Models. Internat. J. Modern Phys. A 19, May, suppl., 276-293 (2004).

[40] Polya, G., Szego, G.: Problems and Theorems in Analysis. Vol.2, Chapter 6, Problem 67. Springer: New-York 1978.

[41] Schlittgen, B., Wettig T.: Generalizations of some integrals over the unitary group. J. Phys. A 36, 3195-3202 (2003).

[42] Shuryak, E.V., Verbaarschot, J.J.M.: Random matrix theory and spectral sum rules for the Dirac operator in QCD. Nucl. Phys. A560, 306-320 (1993).

[43] Strahov, E.: Moments of characteristic polynomials enumerate two-rowed lexicographic arrays. Electronic Journal of Combinatorics, 10, R24 (2003).

[44] Trotter, H.F.: Eigenvalue distributions of large Hermitian matrices: Wigner semicircle and a theorem of Kac, Murdock, and Szego. Adv. Math. 54, 67-82 (1984).

[45] Verbaarschot, J. J. M.: Spectrum of the QCD Dirac Operator and Chiral Random Matrix Theory. Phys. Rev. Lett. 72 , 2531-2533 (1994).

[46] Verbaarschot, J. J. M.: QCD, chiral random matrix theory and integrability. Epreprint arXiv:hep-th/0502029

[47] Wilkinson, J. H.: The Algebraic Eigenvalue Problem, Clarendon Press: Oxford, 1965.

[48] Zirnbauer, M.R.: Supersymmetry for systems with unitary disorder: circular ensembles. J. Phys. A 29, 7113-7136 (1996).

[49] Zyczkowski, K., Sommers, H.-J.: Truncations of random unitary matrices. J. Phys. A 33, 2045-2058 (2000). 